Research Article

\title{
Methods for Solving $L R$-Type Pythagorean Fuzzy Linear Programming Problems with Mixed Constraints
}

\author{
Muhammad Akram $\left(\mathbb{D},{ }^{1}\right.$ Inayat Ullah, ${ }^{1}$ and Majed G. Alharbi ${ }^{2}$ \\ ${ }^{1}$ Department of Mathematics, University of the Punjab, New Campus, Lahore 4590, Pakistan \\ ${ }^{2}$ Department of Mathematics, College of Arts and Sciences, Methnab, Qassim University, Saudi Arabia \\ Correspondence should be addressed to Muhammad Akram; m.akram@pucit.edu.pk
}

Received 23 April 2021; Accepted 21 July 2021; Published 31 July 2021

Academic Editor: Erik Cuevas

Copyright (c) 2021 Muhammad Akram et al. This is an open access article distributed under the Creative Commons Attribution License, which permits unrestricted use, distribution, and reproduction in any medium, provided the original work is properly cited.

\begin{abstract}
A Pythagorean fuzzy set is the superset of fuzzy and intuitionistic fuzzy sets, respectively. Yager proposed the concept of Pythagorean fuzzy sets in which he relaxed the condition that sum of square of both membership degree and nonmembership degree of an element of a set must not be greater than 1 . This paper introduces two new techniques to solve $L R$-type fully Pythagorean fuzzy linear programming problems with mixed constraints having unrestricted LR-type Pythagorean fuzzy numbers as variables and parameters by introducing unknown variables and using a ranking function. Furthermore, we show the equivalence of both the proposed methods and compare the solutions obtained by the two techniques. Besides this, we solve an already existing practical model using proposed techniques and compare the result.
\end{abstract}

\section{Introduction}

The origin of linear programming is the 1940s (World War II). Linear programming is a technique in which a function (called objective function) is an optimized subject to a given set of restrictions (called constraints). It is mostly used in a situation where there is some quantity to be optimized within available resources. The nature of the linear programming model is trivial and easily applicable to various real-life applications, including transportation problems, supplier selections, assignment problems, production planning problems, and supply chain management. Linear programming in a fuzzy environment is a very interesting field in which many researchers showed interest around the globe. It can be used very effectively in the situations, where the data is fuzzy, vague, or uncertain, where crisp theory fails to cope with. Hence, in these situations, fuzzy linear programming technique is very effective in making decisions.

Zadeh [1,2] introduced the concepts of fuzzy sets and fuzzy numbers. Bellman and Zadeh [3] first introduced the concept of decision-making in a fuzzy environment. Zimmermann [4] studied the fuzzy programming technique to solve the multiobjective linear programming problem under a fuzzy environment. The fuzzy optimization technique is based on the maximization of the marginal satisfaction (membership functions and degree of belongingness) of each element into the fuzzy decision set. Tanaka et al. [5] also discussed mathematical linear programming in a fuzzy environment. Allahviranloo [6] presented the Adomian decomposition method for a fuzzy system of linear equations. Allahviranloo et al. [7] discovered a method for solving fully fuzzy linear programming problems by the ranking function. Lotfi et al. [8] presented a method for solving a full fuzzy linear programming using lexicography method and fuzzy approximate solution. Kumar et al. [9] proposed a new method for solving fully fuzzy linear programming problems. Kumar and Kaur [10] studied a method for exact fuzzy optimal solution of fully fuzzy linear programming problems with unrestricted fuzzy variables. Kaur and Kumar [11] presented Mehar's method for solving fully fuzzy linear programming problems with $L R$ fuzzy parameters. Moloudzadeh et al. [12] introduced a new method for solving an arbitrary fully fuzzy linear system. Behera et al. [13] studied new methods for solving 
imprecisely defined linear programming problems under trapezoidal fuzzy uncertainty. Najafi and Edalatpanah [14] introduced a new method for solving fully fuzzy linear programming problems. Pérez-Caňedo et al. [15] gave a revised version of a lexicographical-based method for solving fully fuzzy linear programming problems with inequality constraints.

Later on, it was realized that only the membership degrees are not well enough to represent the marginal attainment of the element into the fuzzy decision set. To extend or explore the fuzzy set, Atanassov [16] introduced the concept of fuzzy set to intuitionistic fuzzy set in which there is a nonmembership function along with the membership function. In an intuitionistic fuzzy set, the sum of membership degree and nonmembership degree of an element should not be greater than 1. Angelov [17] first considered the intuitionistic fuzzy optimization techniques based on intuitionistic fuzzy decision set in decision-making problems. Dubey and Mehra [18] presented linear programming with triangular intuitionistic fuzzy numbers. Parvathi and Malathi [19] proposed a method to solve intuitionistic fuzzy linear programming problems. Nagoorgani and Ponnalagu [20] revealed a new approach on solving intuitionistic fuzzy linear programming problems. Parvathi and Malathi [21] studied intuitionistic fuzzy linear optimization. Parvathi et al. [22] gave intuitionistic fuzzy linear regression analysis. Garg et al. [23] presented an intuitionistic fuzzy optimization technique for solving multiobjective reliability optimization problems in an interval environment. Suresh et al. [24] gave a method of solving intuitionistic fuzzy linear programming problems by ranking function. Nagoorgani et al. [25] presented the knowledge of expert opinion in intuitionistic fuzzy linear programming problems. Singh and Yadav [26] proposed optimization of unrestricted $L R$-type intuitionistic fuzzy mathematical programming problems. Singh and Yadav [27] proposed intuitionistic fuzzy multiobjective linear programming problems with various membership functions. Malathi and Umadevi [28] gave a new procedure for solving linear programming problems in an intuitionistic fuzzy environment. Abhishekh and Nishad [29] proposed a novel ranking approach for solving fully $L R$-intuitionistic fuzzy transportation problem. Bharati and Singh [30] studied a method for the solution of multiobjective linear programming problems in intervalvalued intuitionistic fuzzy environments. Kabiraj et al. [31] proposed another method for intuitionistic fuzzy linear programming problems. Perez-Canedo and ConcepcionMorales [15] presented a method for unique optimal values of $L R$-type fully intuitionistic fuzzy linear programming with inequality constraints.

Unfortunately, intuitionistic fuzzy sets fail to deal with the situations where the sum of membership degree and nonmembership degree of an element exceeds 1 . To overcome this difficulty, Yager [32] introduced the concept of Pythagorean fuzzy set in which he relaxed the condition that sum of square of both membership degree and nonmembership degree of an element of a set must not be greater than 1. Yager and Abbasov [33] presented Pythagorean membership grades, complex numbers, and decision-making. Yager [34] introduced Pythagorean membership grades in multicriteria decision-making. Zhang and $\mathrm{Xu}$ [35] extended the TOPSIS to multiple-criteria decision-making with Pythagorean fuzzy sets. Peng et al. [36] studied Pythagorean fuzzy information measures and their applications. Wan et al. [37] gave a Pythagorean fuzzy mathematical programming method for multiattribute group decisionmaking with Pythagorean fuzzy truth degrees. Kumar et al. [38] proposed a Pythagorean fuzzy approach to the transportation problem. Luqman et al. [39] presented a digraph and matrix approach for risk evaluations under Pythagorean fuzzy information. Wan et al. [40] gave a new order relation for Pythagorean fuzzy numbers and its application to multiattribute group decision-making. On the contrary, Ahmad et al. [41] studied spherical fuzzy linear programming problems and Akram et al. [42] developed methods to solve fully Pythagorean fuzzy linear programming problems with equality constraints. Wei et al. [43] studied green supplier selection based on the CODAS method in a probabilistic uncertain linguistic environment. Wei et al. [44] extended COPRAS model for multiple attribute group decision-making based on single-valued neutrosophic 2tuple linguistic environment. Zhang et al. [45] studied the TODIM method based on cumulative prospect theory for multiple attribute group decision-making under a 2-tuple linguistic Pythagorean fuzzy environment. Recently, Akram et al. [46] have introduced a method to solve linear programming problems in which all the variables and parameters are $L R$-type PFNs having equality constraints. As a continuation of this work, we study two methods for solving FPFLPPs with mixed constraints in which all the variables and parameters are unrestricted $L R$-type Pythagorean fuzzy numbers (PFNs).

The main contribution of this research paper is as follows:

(1) The first method is presented to solve FPFLPPs with mixed constraints, in which all the variables and parameters are $L R$-type PFNs

(2) The second method is presented to handle inequality constraints in FPFLPPs using a ranking function

(3) A comparison of the proposed methods with the existing methods is given

(4) The results of the methods are shown graphically

The article is organized as follows. Section 2 presents some preliminaries. Section 3 explains the proposed methods to solve FPFLPPs with unrestricted LR-type PFNs with mixed constraints. Section 4 presents the equivalence of the proposed techniques. Section 5 is devoted for numerical examples to explain the proposed methods. Section 6 includes comparative analysis and some discussions. In Section 7 , merits of the proposed methods are given. In Section 8, we conclude the paper.

For further information, the reader can refer to [47-57].

\section{Preliminaries}

In this section, we review elementary concepts that are useful for this article. 
Definition 1 (see [34]). A PFS $\Omega$ on $X$ is an object of the form:

$$
\Omega=\left\{\left\langle x, \mu_{\Omega}(x), v_{\Omega}(x)\right\rangle \mid x \in X\right\},
$$

where $\mu_{\mathscr{A}}: X \longrightarrow[0,1]$ and $v_{\mathscr{A}}: X \longrightarrow[0,1]$ are membership function and nonmembership function of $\Omega$, respectively, such that $0 \leq \mu_{\Omega}^{2}(x)+v_{\Omega}^{2}(x) \leq 1, \forall x \in X$. Moreover, for all $x \in X, \pi_{\Omega}(x)=\sqrt{1-\mu_{\Omega}^{2}(x)-v_{\Omega}^{2}(x)}$ is called a Pythagorean fuzzy index or degree of hesitancy of $x$ in $\Omega$. For computational convenience, $\Omega=\left(\mu_{\Omega}, v_{\Omega}\right)$ is called a PFN [35].

Definition 2 (see [46]). A PFN $A=\left(a ; \eta, \theta ; \eta^{\prime}, \theta^{\prime}\right)_{L R}$ is defined as an $L R$-type PFN if its membership $\left(\mu_{A}\right)$ and nonmembership $\left(v_{A}\right)$ functions are given as

$$
\begin{aligned}
& \mu_{A}(x)= \begin{cases}L\left(\frac{a-x}{\eta}\right), & x \leq a, \eta>0, \\
R\left(\frac{x-a}{\theta}\right), & x \geq a, \theta>0,\end{cases} \\
& v_{A}(x)= \begin{cases}L^{\prime}\left(\frac{a-x}{\eta^{\prime}}\right), & x \leq a, \eta^{\prime}>0, \\
R^{\prime}\left(\frac{x-a}{\theta^{\prime}}\right), & x \geq a, \theta^{\prime}>0,\end{cases}
\end{aligned}
$$

where $\eta \leq \eta^{\prime}, \theta \leq \theta^{\prime}$ and $0 \leq \mu_{A}^{2}+v_{A}^{2} \leq 1$. $L$ and $R$ are continuous, decreasing functions on $[0, \infty)$ and $L^{\prime}$ and $R^{\prime}$ are continuous increasing functions on $[0, \infty)$ such that

(1) $L(0)=R(0)=1$

(2) $\lim _{x \rightarrow \infty} R(x)=\lim _{x \rightarrow \infty} L(x)=0$

(3) $L^{\prime}(0)=R^{\prime}(0)=0$

(4) $\lim _{x \longrightarrow \infty} R^{\prime}(x)=\lim _{x \longrightarrow \infty} L^{\prime}(x)=1$

$a$ is called the mean value of $A, \eta$ and $\theta$ are the left and right spreads of $\mu_{A}$, and $\eta^{\prime}$ and $\theta^{\prime}$ are left and right spreads of $\nu_{A}$, respectively.

Remark 1 (see [46]).

(1) If we set $L^{\prime}(x)=1-L(x)$ and $R^{\prime}(x)=1-R(x)$ in Definition 2, then $A=\left(a ; \eta, \theta ; \eta^{\prime}, \theta^{\prime}\right)_{L R}$ becomes $L R$-type intuitionistic fuzzy number [26].

(2) If we take

$$
\begin{gathered}
L(x)=R(x)= \begin{cases}1-x, & 0 \leq x \leq 1, \\
0, & \text { otherwise }\end{cases} \\
L^{\prime}(x)=R^{\prime}(x)= \begin{cases}x, & 0 \leq x \leq 1, \\
1, & \text { otherwise }\end{cases}
\end{gathered}
$$

in Definition 2, then $A=\left(a ; \eta, \theta ; \eta^{\prime}, \theta^{\prime}\right)_{L R}$ becomes triangular PFN.

Definition 3 (see [46]). An LR-type PFN $A=(a ; \eta, \theta$; $\left.\eta^{\prime}, \theta^{\prime}\right)_{L R}$ is nonnegative (respectively, nonpositive), denoted as $A \geq 0$ (respectively, $A \leq 0$ ), if $a-\eta^{\prime} \geq 0$ (respectively $a+\theta^{\prime}$ $\leq 0)$ and $A$ is unrestricted if $a$ belongs to real numbers.

Definition 4 (see [46]). An LR-type PFN $A=\left(a ; \eta, \theta ; \eta^{\prime}\right.$, $\left.\theta^{\prime}\right)_{L R}$ is positive if $a-\eta^{\prime}>0$ and negative if $a+\theta^{\prime}<0$.

Definition 5 (see [46]). An $L R$-type PFN $A=\left(a ; \eta, \theta ; \eta^{\prime}\right.$, $\left.\theta^{\prime}\right)_{L R}$ is zero if and only if $a=0, \eta=0, \theta=0, \eta^{\prime}=0$, and $\theta^{\prime}=0$.

Definition 6 (see [46]). Two LR-type PFNs $A_{1}=$ $\left(a_{1} ; \eta_{1}, \theta_{1} ; \eta_{1}^{\prime}, \theta_{1}^{\prime}\right)_{L R}$ and $A_{2}=\left(a_{2} ; \eta_{2}, \theta_{2} ; \eta_{2}^{\prime}, \theta_{2}^{\prime}\right)_{L R}$ are equal if $a_{1}=a_{2}, \eta_{1}=\eta_{2}, \theta_{1}=\theta_{2}, \eta_{1}^{\prime}=\eta_{2}^{\prime}$, and $\theta_{1}^{\prime}=\theta_{2}^{\prime}$.

Theorem 1 (see [46]). Let $A_{1}=\left(a_{1} ; \eta_{1}, \theta_{1} ; \eta_{1}^{\prime}, \theta_{1}^{\prime}\right)_{L R}$ and $A_{2}=\left(a_{2} ; \eta_{2}, \theta_{2} ; \eta_{2}^{\prime}, \theta_{2}^{\prime}\right)_{L R}$ be two LR-type PFNs; then, $A_{1} \oplus A_{2}=\left(a_{1}+a_{2} ; \eta_{1}+\eta_{2}, \theta_{1}+\theta_{2} ; \eta_{1}^{\prime}+\eta_{2}^{\prime}, \theta_{1}^{\prime}+\theta_{2}^{\prime}\right)_{L R}$.

Theorem 2 (see [46]). Let $A_{1}=\left(a_{1} ; \eta_{1}, \theta_{1} ; \eta_{1}^{\prime}, \theta_{1}^{\prime}\right)_{L R}$ and $A_{2}=\left(a_{2} ; \eta_{2}, \theta_{2} ; \eta_{2}^{\prime}, \theta_{2}^{\prime}\right)_{L R}$ be two LR-type PFNs; then, $A_{1} \ominus A_{2}=\left(a_{1}-a_{2} ; \eta_{1}+\theta_{2}, \theta_{1}+\eta_{2} ; \eta_{1}^{\prime}+\theta_{2}^{\prime}, \theta_{1}^{\prime}+\eta_{2}^{\prime}\right)_{L R}$

Theorem 3 (see [46]). Let $A=\left(\left(a ; \eta, \theta ; \eta^{\prime}, \theta^{\prime}\right)_{L R}\right.$ be an LR-type PFN and $c$ be any real number; then,

$$
c A= \begin{cases}\left(c a ; c \eta, c \theta ; c \eta^{\prime}, c \theta^{\prime}\right)_{L R}, & c \geq 0, \\ \left(c a ;-c \theta,-c \eta ;-c \theta^{\prime},-c \eta^{\prime}\right)_{L R}, & c<0 .\end{cases}
$$

Theorem 4 (see [46]). Let $A_{1}=\left(a_{1} ; \eta_{1}, \theta_{1} ; \eta_{1}^{\prime}, \theta_{1}^{\prime}\right)_{L R}$ and $A_{2}=\left(a_{2} ; \eta_{2}, \theta_{2} ; \eta_{2}^{\prime}, \theta_{2}^{\prime}\right)_{L R}$ be two nonnegative LR-type PFNs; then, $A_{1} \otimes A_{2}=\left(a_{1} a_{2} ; a_{1} \eta_{2}+a_{2} \eta_{1}-\eta_{1} \eta_{2}, a_{1} \theta_{2}+a_{2} \theta_{1}+\right.$ $\left.\theta_{1} \theta_{2} ; a_{1} \eta_{2}^{\prime}+a_{2} \eta_{1}^{\prime}-\eta_{1}^{\prime} \eta_{2}^{\prime}, a_{1} \theta_{2}^{\prime}+a_{2} \theta_{1}^{\prime}+\theta_{1}^{\prime} \theta_{2}^{\prime}\right)_{L R}$.

Theorem 5 (see [46]). Let $A_{1}=\left(a_{1} ; \eta_{1}, \theta_{1} ; \eta_{1}^{\prime}, \theta_{1}^{\prime}\right)_{L R}$ be nonnegative LR-type PFN and $A_{2}=\left(a_{2} ; \eta_{2}, \theta_{2} ; \eta_{2}^{\prime}, \theta_{2}^{\prime}\right)_{L R}$ be nonpositive LR-type PFN; then, $A_{1} \otimes A_{2}=\left(a_{1} a_{2} ; a_{1}\right.$ $\eta_{2}-a_{2} \theta_{1}+\eta_{2} \theta_{1}, a_{1} \theta_{2}-a_{2} \eta_{1}-\eta_{1} \theta_{2} ; a_{1} \eta_{2}^{\prime}-a_{2} \theta_{1}{ }^{\prime}+\eta_{2}^{\prime} \theta_{1}^{\prime}, a_{1} \theta_{2}^{\prime}$ $\left.-a_{2} \eta_{1}^{\prime}-\eta_{1}^{\prime} \theta_{2}^{\prime}\right)_{L R}$

Theorem 6 (see [46]). Let $A_{1}=\left(a_{1} ; \eta_{1}, \theta_{1} ; \eta_{1}^{\prime}, \theta_{1}^{\prime}\right)_{L R}$ be an LR-type PFN in which $a_{1}-\eta_{1}^{\prime}<0, a_{1}-\eta_{1} \geq 0$ and $A_{2}=\left(a_{2} ; \eta_{2}, \theta_{2} ; \eta_{2}^{\prime}, \theta_{2}^{\prime}\right)_{L R}$ be an unrestricted LR-type PFN; then, $A_{1} \otimes A_{2}=\left(a ; \eta, \theta ; \eta^{\prime}, \theta^{\prime}\right)_{L R}$, where $a=a_{1} a_{2}$ : 


$$
\begin{aligned}
\eta & =a_{1} a_{2}-\min \left\{a_{1} a_{2}-\eta_{2} a_{1}-\eta_{1} a_{2}+\eta_{1} \eta_{2}, a_{1} a_{2}-\eta_{2} a_{1}+\theta_{1} a_{2}-\eta_{2} \theta_{1}\right\} \\
\theta & =\max \left\{a_{1} a_{2}+\theta_{2} a_{1}+\theta_{1} a_{2}+\theta_{1} \theta_{2}, a_{1} a_{2}+\theta_{2} a_{1}-\eta_{1} a_{2}-\eta_{1} \theta_{2}\right\}-a_{1} a_{2}, \\
\eta^{\prime} & =a_{1} a_{2}-\min \left\{a_{1} a_{2}-\eta_{1}^{\prime} a_{2}+\theta_{2}^{\prime} a_{1}-\eta_{1}^{\prime} \theta_{2}^{\prime}, a_{1} a_{2}+\theta_{1}^{\prime} a_{2}-\eta_{2}^{\prime} a_{1}-\eta_{2}^{\prime} \theta_{1}^{\prime}\right\} \\
\theta^{\prime} & =\max \left\{a_{1} a_{2}-\eta_{1}^{\prime} a_{2}-\eta_{2}^{\prime} a_{1}+\eta_{1}^{\prime} \eta_{2}^{\prime}, a_{1} a_{2}+\theta_{1}^{\prime} a_{2}+\theta_{2}^{\prime} a_{1}+\theta_{1}^{\prime} \theta_{2}^{\prime}\right\}-a_{1} a_{2} .
\end{aligned}
$$

Theorem 7 (see [46]). Let $A_{1}=\left(a_{1} ; \eta_{1}, \theta_{1} ; \eta_{1}^{\prime}, \theta_{1}^{\prime}\right)_{L R}$ be an LR-type PFN in which $a_{1}-\eta_{1}<0, \quad a_{1} \geq 0$ and
$A_{2}=\left(a_{2} ; \eta_{2}, \theta_{2} ; \eta_{2}^{\prime}, \theta_{2}^{\prime}\right)_{L R}$ be an unrestricted LR-type PFN; then, $A_{1} \otimes A_{2}=\left(a ; \eta, \theta ; \eta^{\prime}, \theta^{\prime}\right)_{L R}$, where $a=a_{1} a_{2}$ :

$$
\begin{aligned}
\eta & =a_{1} a_{2}-\min \left\{a_{1} a_{2}-\eta_{1} a_{2}+\theta_{2} a_{1}-\eta_{1} \theta_{2}, a_{1} a_{2}+\theta_{1} a_{2}-\eta_{2} a_{1}-\eta_{2} \theta_{1}\right\}, \\
\theta & =\max \left\{a_{1} a_{2}-\eta_{1} a_{2}-\eta_{2} a_{1}+\eta_{1} \eta_{2}, a_{1} a_{2}+\theta_{1} a_{2}+\theta_{2} a_{1}+\theta_{1} \theta_{2}\right\}-a_{1} a_{2}, \\
\eta^{\prime} & =a_{1} a_{2}-\min \left\{a_{1} a_{2}-\eta_{1}^{\prime} a_{2}+\theta_{2}^{\prime} a_{1}-\eta_{1}^{\prime} \theta_{2}^{\prime}, a_{1} a_{2}+\theta_{1}^{\prime} a_{2}-\eta_{2}^{\prime} a_{1}-\eta_{2}^{\prime} \theta_{1}^{\prime}\right\} \\
\theta^{\prime} & =\max \left\{a_{1} a_{2}-\eta_{1}^{\prime} a_{2}-\eta_{2}^{\prime} a_{1}+\eta_{1}^{\prime} \eta_{2}^{\prime}, a_{1} a_{2}+\theta_{1}^{\prime} a_{2}+\theta_{2}^{\prime} a_{1}+\theta_{1}^{\prime} \theta_{2}^{\prime}\right\}-a_{1} a_{2} .
\end{aligned}
$$

Theorem 8 (see [46]). Let $A_{1}=\left(a_{1} ; \eta_{1}, \theta_{1} ; \eta_{1}^{\prime}, \theta_{1}^{\prime}\right)_{L R}$ be an LR-type PFN in which $a_{1}<0, \quad a_{1}+\theta_{1} \geq 0$ and
$A_{2}=\left(a_{2} ; \eta_{2}, \theta_{2} ; \eta_{2}^{\prime}, \theta_{2}^{\prime}\right)_{L R}$ be an unrestricted LR-type PFN; then, $A_{1} \otimes A_{2}=\left(a ; \eta, \theta ; \eta^{\prime}, \theta^{\prime}\right)_{L R}$, where $a=a_{1} a_{2}$ :

$$
\begin{aligned}
\eta & =a_{1} a_{2}-\min \left\{a_{1} a_{2}-\eta_{1} a_{2}+\theta_{2} a_{1}-\eta_{1} \theta_{2}, a_{1} a_{2}+\theta_{1} a_{2}-\eta_{2} a_{1}-\eta_{2} \theta_{1}\right\} \\
\theta & =\max \left\{a_{1} a_{2}-\eta_{1} a_{2}-\eta_{2} a_{1}+\eta_{1} \eta_{2}, a_{1} a_{2}+\theta_{1} a_{2}+\theta_{2} a_{1}+\theta_{1} \theta_{2}\right\}-a_{1} a_{2}, \\
\eta^{\prime} & =a_{1} a_{2}-\min \left\{a_{1} a_{2}-\eta_{1}^{\prime} a_{2}+\theta_{2}^{\prime} a_{1}-\eta_{1}^{\prime} \theta_{2}^{\prime}, a_{1} a_{2}+\theta_{1}^{\prime} a_{2}-\eta_{2}^{\prime} a_{1}-\eta_{2}^{\prime} \theta_{1}^{\prime}\right\}, \\
\theta^{\prime} & =\max \left\{a_{1} a_{2}-\eta_{1}^{\prime} a_{2}-\eta_{2}^{\prime} a_{1}+\eta_{1}^{\prime} \eta_{2}^{\prime}, a_{1} a_{2}+\theta_{1}^{\prime} a_{2}+\theta_{2}^{\prime} a_{1}+\theta_{1}^{\prime} \theta_{2}^{\prime}\right\}-a_{1} a_{2} .
\end{aligned}
$$

Theorem 9 (see [46]). Let $A_{1}=\left(a_{1} ; \eta_{1}, \theta_{1} ; \eta_{1}^{\prime}, \theta_{1}^{\prime}\right)_{L R}$ be an LR-type PFN in which $a_{1}+\theta_{1}<0, a_{1}+\theta_{1}^{\prime} \geq 0$ and
$A_{2}=\left(a_{2} ; \eta_{2}, \theta_{2} ; \eta_{2}^{\prime}, \theta_{2}^{\prime}\right)_{L R}$ be an unrestricted LR-type PFN; then, $A_{1} \otimes A_{2}=\left(a ; \eta, \theta ; \eta^{\prime}, \theta^{\prime}\right)_{L R}$, where $a=a_{1} a_{2}$ :

$$
\begin{aligned}
\eta & =a_{1} a_{2}-\min \left\{a_{1} a_{2}-\eta_{1} a_{2}+\theta_{2} a_{1}-\eta_{1} \theta_{2}, a_{1} a_{2}+\theta_{2} a_{1}+\theta_{1} a_{2}+\theta_{1} \theta_{2}\right\} \\
\theta & =\max \left\{a_{1} a_{2}+\theta_{1} a_{2}-\eta_{2} a_{1}-\eta_{2} \theta_{1}, a_{1} a_{2}-\eta_{2} a_{1}-\eta_{1} a_{2}+\eta_{1} \eta_{2}\right\}-a_{1} a_{2}, \\
\eta^{\prime} & =a_{1} a_{2}-\min \left\{a_{1} a_{2}-\eta_{1}^{\prime} a_{2}+\theta_{2}^{\prime} a_{1}-\eta_{1}^{\prime} \theta_{2}^{\prime}, a_{1} a_{2}+\theta_{1}^{\prime} a_{2}-\eta_{2}^{\prime} a_{1}-\eta_{2}^{\prime} \theta_{1}^{\prime}\right\} \\
\theta^{\prime} & =\max \left\{a_{1} a_{2}-\eta_{1}^{\prime} a_{2}-\eta_{2}^{\prime} a_{1}+\eta_{1}^{\prime} \eta_{2}^{\prime}, a_{1} a_{2}+\theta_{1}^{\prime} a_{2}+\theta_{2}^{\prime} a_{1}+\theta_{1}^{\prime} \theta_{2}^{\prime}\right\}-a_{1} a_{2} .
\end{aligned}
$$

Theorem 10 (see [46]). Let $A_{1}=\left(a_{1} ; \eta_{1}, \theta_{1} ; \eta_{1}^{\prime}, \theta_{1}^{\prime}\right)_{L R}$ be an LR-type PFN in which $a_{1}+\theta_{1}^{\prime}<0$ and
$A_{2}=\left(a_{2} ; \eta_{2}, \theta_{2} ; \eta_{2}^{\prime}, \theta_{2}^{\prime}\right)_{L R}$ be an unrestricted LR-type PFN; then, $A_{1} \otimes A_{2}=\left(a ; \eta, \theta ; \eta^{\prime}, \theta^{\prime}\right)_{L R}$, where $a=a_{1} a_{2}$ :

$$
\begin{aligned}
\eta & =a_{1} a_{2}-\min \left\{a_{1} a_{2}-\eta_{1} a_{2}+\theta_{2} a_{1}-\eta_{1} \theta_{2}, a_{1} a_{2}+\theta_{2} a_{1}+\theta_{1} a_{2}+\theta_{1} \theta_{2}\right\}, \\
\theta & =\max \left\{a_{1} a_{2}+\theta_{1} a_{2}-\eta_{2} a_{1}-\eta_{2} \theta_{1}, a_{1} a_{2}-\eta_{2} a_{1}-\eta_{1} a_{2}+\eta_{1} \eta_{2}\right\}-a_{1} a_{2}, \\
\eta^{\prime} & =a_{1} a_{2}-\min \left\{a_{1} a_{2}-\eta_{1}^{\prime} a_{2}+\theta_{2}^{\prime} a_{1}-\eta_{1}^{\prime} \theta_{2}^{\prime}, a_{1} a_{2}+\theta_{2}^{\prime} a_{1}+\theta_{1}^{\prime} a_{2}+\theta_{1}^{\prime} \theta_{2}^{\prime}\right\}, \\
\theta^{\prime} & =\max \left\{a_{1} a_{2}+\theta_{1}^{\prime} a_{2}-\eta_{2}^{\prime} a_{1}-\eta_{2}^{\prime} \theta_{1}^{\prime}, a_{1} a_{2}-\eta_{1}^{\prime} a_{2}-\eta_{2}^{\prime} a_{1}+\eta_{1}^{\prime} \eta_{2}^{\prime}\right\}-a_{1} a_{2} .
\end{aligned}
$$

Theorem 11 (see [46]). Let $A_{1}=\left(a_{1} ; \eta_{1}, \theta_{1} ; \eta_{1}^{\prime}, \theta_{1}^{\prime}\right)_{L R}$ be an LR-type PFN in which $a_{1}-\eta_{1}^{\prime} \geq 0$ and
$A_{2}=\left(a_{2} ; \eta_{2}, \theta_{2} ; \eta_{2}^{\prime} \theta_{2}^{\prime}\right)_{L R}$ be an unrestricted LR-type PFN; then, $A_{1} \otimes A_{2}=\left(a ; \eta, \theta ; \eta^{\prime}, \theta^{\prime}\right)_{L R}$, where $a=a_{1} a_{2}$ : 


$$
\begin{aligned}
\eta & =a_{1} a_{2}-\min \left\{a_{1} a_{2}-\eta_{2} a_{1}-\eta_{1} a_{2}+\eta_{1} \eta_{2}, a_{1} a_{2}-\eta_{2} a_{1}+\theta_{1} a_{2}-\eta_{2} \theta_{1}\right\} \\
\theta & =\max \left\{a_{1} a_{2}+\theta_{2} a_{1}+\theta_{1} a_{2}+\theta_{1} \theta_{2}, a_{1} a_{2}+\theta_{2} a_{1}-\eta_{1} a_{2}-\eta_{1} \theta_{2}\right\}-a_{1} a_{2}, \\
\eta^{\prime} & =a_{1} a_{2}-\min \left\{a_{1} a_{2}-\eta_{2}^{\prime} a_{1}-\eta_{1}^{\prime} a_{2}+\eta_{1}^{\prime} \eta_{2}^{\prime}, a_{1} a_{2}-\eta_{2}^{\prime} a_{1}+\theta_{1}^{\prime} a_{2}-\eta_{2}^{\prime} \theta_{1}^{\prime}\right\}, \\
\theta^{\prime} & =\max \left\{a_{1} a_{2}+\theta_{2}^{\prime} a_{1}+\theta_{1}^{\prime} a_{2}+\theta_{1}^{\prime} \theta_{2}^{\prime}, a_{1} a_{2}+\theta_{2}^{\prime} a_{1}-\eta_{1}^{\prime} a_{2}-\eta_{1}^{\prime} \theta_{2}^{\prime}\right\}-a_{1} a_{2} .
\end{aligned}
$$

Definition 7 (see [46]). Let $A=\left(a ; \eta, \theta ; \eta^{\prime}, \theta^{\prime}\right)_{L R}$ be an $L R$-type PFN; then, ranking of $A$, denoted $\mathfrak{R}(A)$, can be defined as

$\mathfrak{R}(A)=\frac{1}{4}\left[\left(\int_{0}^{1}\left(a-\eta L^{-1}(\alpha)\right) \mathrm{d} \alpha+\int_{0}^{1}\left(a+\theta R^{-1}(\alpha)\right) \mathrm{d} \alpha\right)+\left(\int_{0}^{1}\left(a-\eta^{\prime} L^{\prime-1}(\alpha)\right) \mathrm{d} \alpha+\int_{0}^{1}\left(a+\theta^{\prime} R^{\prime-1}(\alpha)\right) \mathrm{d} \alpha\right)\right]$.

Let $A_{1}$ and $A_{2}$ be two $L R$-type PFN; then, we see that

(i) $A_{1} \preccurlyeq A_{2}$ if $\mathfrak{R}\left(A_{1}\right)<\mathfrak{R}\left(A_{2}\right)$

(ii) $A_{1} \geqslant A_{2}$ if $\mathfrak{R}\left(A_{1}\right)>\mathfrak{R}\left(A_{2}\right)$

(iii) $A_{1} \approx A_{2}$ if $\mathfrak{R}\left(A_{1}\right)=\mathfrak{R}\left(A_{2}\right)$
Remark 2 (see [46]). Ranking function, as defined in Definition 7 , is a linear function.

Proof. Let $A_{1}=\left(a_{1} ; \eta_{1}, \theta_{1} ; \eta_{1}^{\prime}, \theta_{1}^{\prime}\right)_{L R}$ and $A_{2}=\left(a_{2} ; \eta_{2}, \theta_{2} ; \eta_{2}^{\prime}\right.$, $\left.\theta_{2}^{\prime}\right)_{L R}$ be two LR-type PFNs; then, $A_{1} \oplus A_{2}=\left(a_{1}+a_{2} ; \eta_{1}+\right.$ $\left.\eta_{2}, \theta_{1}+\theta_{2} ; \eta_{1}^{\prime}+\eta_{2}^{\prime}, \theta_{1}^{\prime}+\theta_{2}^{\prime}\right)_{L R}$.

Now, if $L=R$,

$$
\begin{aligned}
\Re\left(A_{1} \oplus A_{2}\right)= & \operatorname{Re}\left(a_{1}+a_{2} ; \eta_{1}+\eta_{2}, \theta_{1}+\theta_{2} ; \eta_{1}^{\prime}+\eta_{2}^{\prime}, \theta_{1}^{\prime}+\theta_{2}^{\prime}\right)_{L R} \\
= & \frac{1}{4}\left[\left(\int_{0}^{1}\left(a_{1}+a_{2}-\left(\eta_{1}+\eta_{2}\right) L^{-1}(\alpha)\right) \mathrm{d} \alpha+\int_{0}^{1}\left(a_{1}+a_{2}+\left(\theta_{1}+\theta_{2}\right) R^{-1}(\alpha)\right) \mathrm{d} \alpha\right)\right. \\
& \left.+\left(\int_{0}^{1}\left(a_{1}+a_{2}-\left(\eta_{1}^{\prime}+\eta_{2}^{\prime}\right) L^{\prime-1}(\alpha)\right) \mathrm{d} \alpha+\int_{0}^{1}\left(a_{1}+a_{2}+\left(\theta_{1}^{\prime}+\theta_{2}^{\prime}\right) R^{\prime-1}(\alpha)\right) \mathrm{d} \alpha\right)\right] \\
= & \frac{1}{4}\left[\left(\int_{0}^{1}\left(a_{1}-\eta_{1} L^{-1}(\alpha)\right) \mathrm{d} \alpha+\int_{0}^{1}\left(a_{1}+\theta_{1} R^{-1}(\alpha)\right) \mathrm{d} \alpha\right)+\left(\int_{0}^{1}\left(a_{1}-\eta_{1}^{\prime} L^{\prime-1}(\alpha)\right) \mathrm{d} \alpha\right.\right. \\
& \left.\left.+\int_{0}^{1}\left(a_{1}+\theta_{1}^{\prime} R^{\prime-1}(\alpha)\right) \mathrm{d} \alpha\right)\right]+\frac{1}{4}\left[\left(\int_{0}^{1}\left(a_{2}-\eta_{2} L^{-1}(\alpha)\right) \mathrm{d} \alpha+\int_{0}^{1}\left(a_{2}+\theta_{2} R^{-1}(\alpha)\right) \mathrm{d} \alpha\right)\right. \\
& \left.+\left(\int_{0}^{1}\left(a_{2}-\eta_{2}^{\prime} L^{\prime-1}(\alpha)\right) \mathrm{d} \alpha+\int_{0}^{1}\left(a_{2}+\theta_{2}^{\prime} R^{\prime-1}(\alpha)\right) \mathrm{d} \alpha\right)\right] \\
= & \Re\left(A_{1}\right)+\Re\left(A_{2}\right) . \\
& \left.\Re \eta_{1}, \theta_{1} ; \eta_{1}^{\prime}, \theta_{1}^{\prime}\right)_{L R}+\Re\left(a_{2} ; \eta_{2}, \theta_{2} ; \eta_{2}^{\prime}, \theta_{2}^{\prime}\right)_{L R} \\
& \Re
\end{aligned}
$$


Similarly, for some scalar $c, \mathfrak{R}\left(c A_{1}\right)=c \Re\left(A_{1}\right)$.

Hence, ranking function, as defined in Definition 7 , is a linear function.

\section{Methodology to Solve $L R$-Type Fully Pythagorean Fuzzy Linear Programming Problems}

We state here our proposed FPFLPP with $L R$-type PFNs as

$$
\operatorname{Max} / \operatorname{Min} \sum_{j=1}^{n} C_{j} \otimes X_{j},
$$

which subject to

$$
\sum_{j=1}^{n} A_{i j} \otimes X_{j} \preccurlyeq,=, \geqslant B_{i}, \quad \forall i=1,2, \ldots, m,
$$

where $A_{i j}, X_{j}, B_{i}$, and $C_{j}$ are $L R$-type PFNs.

3.1. Method 1: FPFLPP Using Unknown Variables. Here, we state a criterion for the optimal solution of FPFLPP (13).

Definition 8. An LR-type Pythagorean fuzzy optimal solution of FPFLPP (13) with LR-type PFNs will be LR-type PFNs $X_{j}$ if

(1) $X_{j}$ are $L R$-type PFNs

(2) $\sum_{j=1}^{n} A_{i j} \otimes X_{j} \oplus S_{i}=B_{i} \oplus S_{i}^{\prime}, \quad \forall i=1,2, \ldots, m$, such that $\sum_{j=1}^{n} A_{i j} \otimes X_{j} \geqslant B_{i}$ for some PFNs $S_{i}$ and $S_{i}^{\prime}$ satisfying $\quad \mathfrak{R}\left(S_{i}\right)-\mathfrak{R}\left(S_{i}^{\prime}\right) \geq 0 ; \quad \sum_{j=1}^{n} A_{i j} \otimes X_{j} \oplus S_{i}=$ $B_{i} \oplus S_{i}^{\prime}, \forall i=1,2, \ldots, m$, such that $\sum_{j=1}^{n} A_{i j} \otimes X_{j} \geqslant B_{i}$ for some PFNs $S_{i}$ and $S_{i}^{\prime}$ satisfying $\mathfrak{R}\left(S_{i}\right)-\mathfrak{R}\left(S_{i}^{\prime}\right) \leq 0$; and $\sum_{j=1}^{n} A_{i j} \otimes X_{j}=B_{i}, \forall i=1,2, \ldots, m$, such that $\sum_{j=1}^{n} A_{i j} \otimes X_{j}=B_{i}$

(3) If there exist any $L R$-type PFNs $X_{j}^{\prime}$ satisfying step 2, then $\mathfrak{R}\left(\sum_{j=1}^{n} C_{j} \otimes X_{j}\right)>\mathfrak{R}\left(\sum_{j=1}^{n} C_{j} \otimes X_{j}^{\prime}\right)$ in maximization problem and $\mathfrak{R}\left(\sum_{j=1}^{n} C_{j} \otimes X_{j}\right)<\mathfrak{R}\left(\sum_{j=1}^{n}\right.$ $\left.C_{j} \otimes X_{j}^{\prime}\right)$ in minimization problem

The statement of our proposed problem is given in equation (13). We now present steps to solve proposed FPFLPP (13).

Step 1: separating all the constraints into three categories, $\quad \sum_{j=1}^{n} A_{l j} \otimes X_{j} \preccurlyeq B_{l}, \forall l \in M_{1}, \quad \sum_{j=1}^{n} A_{r j} \otimes X_{j}=$ $B_{r}, \forall r \in M_{2}$ and $\sum_{j=1}^{n} A_{s j} \otimes X_{j} \geqslant B_{s}, \forall s \in M_{3}$, the FPFLPP (13) can be rewritten as

$$
\operatorname{Max} / \operatorname{Min} \sum_{j=1}^{n} C_{j} \otimes X_{j} \text {, }
$$

which subject to

$$
\begin{aligned}
& \sum_{j=1}^{n} A_{l j} \otimes X_{j} \preccurlyeq B_{l}, \quad \forall l \in M_{1}, \\
& \sum_{j=1}^{n} A_{r j} \otimes X_{j}=B_{r}, \quad \forall r \in M_{2}, \\
& \sum_{j=1}^{n} A_{s j} \otimes X_{j} \geqslant B_{s}, \quad \forall s \in M_{3},
\end{aligned}
$$

where $X_{j}$ are LR-type PFNs and $M_{1}=$ $\left\{i: 1 \leq i \leq m, \sum_{j=1}^{n} A_{i j} \otimes X_{j} \preccurlyeq B_{i}\right\}, \quad M_{2}=\{i: \quad 1 \leq i \leq m$, $\left.\sum_{j=1}^{n} A_{i j} \otimes X_{j}=B_{i}\right\}$, and $M_{3}=\left\{i: 1 \leq i \leq m, \sum_{j=1}^{n} A_{i j} \otimes\right.$ $\left.X_{j} \geqslant B_{i}\right\}$.

Step 2: introduce the variable $S_{l}$ on left side and $S_{l}^{\prime}$ on right side of the inequality constraint $\sum_{j=1}^{n} A_{l j} \otimes X_{j} \preccurlyeq B_{l}, \forall l \in M_{1}$, to convert it into equality constraint as below:

$$
\sum_{j=1}^{n} A_{l j} \otimes X_{j} \oplus S_{l}=B_{l} \oplus S_{l}^{\prime}, \quad \forall l \in M_{1},
$$

where $\mathfrak{R}\left(S_{l}\right)-\mathfrak{R}\left(S_{l}^{\prime}\right) \geq 0$.

Introduce the variable $S_{s}$ on left side and $S_{s}^{\prime}$ on right side of the inequality constraint $\sum_{j=1}^{n} A_{s j} \otimes X_{j} \geqslant$ $B_{s}, \forall s \in M_{3}$, to convert it into equality constraint as below:

$$
\sum_{j=1}^{n} A_{s j} \otimes X_{j} \oplus S_{s}=B_{s} \oplus S_{s}^{\prime}, \quad \forall s \in M_{3},
$$

where $\mathfrak{R}\left(S_{s}\right)-\mathfrak{R}\left(S_{s}^{\prime}\right) \leq 0$.

The FPFLPP (15) can be written as

$$
\operatorname{Max} / \operatorname{Min} \sum_{j=1}^{n} C_{j} \otimes X_{j},
$$

which subject to

$$
\begin{gathered}
\sum_{j=1}^{n} A_{l j} \otimes X_{j} \oplus S_{l}=B_{l} \oplus S_{l}^{\prime}, \quad \forall l \in M_{1}, \\
\sum_{j=1}^{n} A_{s j} \otimes X_{j} \oplus S_{s}=B_{s} \oplus S_{s}^{\prime}, \quad \forall s \in M_{3}, \\
\sum_{j=1}^{n} A_{r j} \otimes X_{j}=B_{r}, \quad \forall r \in M_{2}, \\
\mathfrak{R}\left(S_{l}\right)-\mathfrak{R}\left(S_{l}^{\prime}\right) \geq 0, \quad \forall l \in M_{1}, \\
\mathfrak{R}\left(S_{s}\right)-\mathfrak{R}\left(S_{s}^{\prime}\right) \leq 0, \quad \forall s \in M_{3},
\end{gathered}
$$

where $X_{j}, S_{l}, S_{l}^{\prime}, S_{s}$, and $S_{s}^{\prime}$ are $L R$-type PFNs.

Step 3: by assuming $A_{i j}=\left(a_{i j} ; \rho_{i j}, \tau_{i j} ; \rho_{i j}^{\prime}, \tau_{i j}^{\prime}\right)_{L R}$, $X_{j}=\left(x_{j} ; \alpha_{j}, \beta_{j} ; \alpha_{j}^{\prime}, \beta_{j}^{\prime}\right)_{L R}, B_{i}=\left(b_{i} ; \theta_{i}, \phi_{i} ; \theta_{i}^{\prime}, \phi_{i}^{\prime}\right)_{L R}, C_{j}=$ 
$\left(c_{j} ; \zeta_{j}, \eta_{j} ; \zeta_{j}^{\prime}, \eta_{j}^{\prime}\right)_{L R}, S_{i}=\left(m_{i} ; e_{i}, f_{i} ; g_{i}, h_{i}\right)_{L R}$, and $S_{i}^{\prime}=$ which subject to $\left(m_{i}^{\prime} ; e_{i}^{\prime}, f_{i}^{\prime} ; g_{i}^{\prime}, h_{i}^{\prime}\right)_{L R}$, the FPFLPP (19) can be rewritten as $\operatorname{Max} / \operatorname{Min} \sum_{j=1}^{n}\left(c_{j} ; \zeta_{j}, \eta_{j} ; \zeta_{j}^{\prime}, \eta_{j}^{\prime}\right)_{L R} \otimes\left(x_{j} ; \alpha_{j}, \beta_{j} ; \alpha_{j}^{\prime}, \beta_{j}^{\prime}\right)_{L R^{\prime}}$

$$
\begin{aligned}
\sum_{j=1}^{n} & \left(a_{l j} ; \rho_{l j}, \tau_{l j} ; \rho_{l j}^{\prime}, \tau_{l j}^{\prime}\right)_{L R} \otimes\left(x_{j} ; \alpha_{j}, \beta_{j} ; \alpha_{j}^{\prime}, \beta_{j}^{\prime}\right)_{L R} \oplus\left(m_{l} ; e_{l}, f_{l} ; g_{l}, h_{l}\right)_{L R} \\
= & \left(b_{l} ; \theta_{l}, \phi_{l} ; \theta_{l}^{\prime}, \phi_{l}^{\prime}\right)_{L R} \oplus\left(m_{l}^{\prime} ; e_{l}^{\prime}, f_{l}^{\prime} ; g_{l}^{\prime}, h_{l}^{\prime}\right)_{L R}, \quad \forall l \in M_{1}, \\
& \sum_{j=1}^{n}\left(a_{s j} ; \rho_{s j}, \tau_{s j} ; \rho_{s j}^{\prime}, \tau_{s j}^{\prime}\right)_{L R} \otimes\left(x_{j} ; \alpha_{j}, \beta_{j} ; \alpha_{j}^{\prime}, \beta_{j}^{\prime}\right)_{L R} \oplus\left(m_{s} ; e_{s}, f_{s} ; g_{s}, h_{s}\right)_{L R} \\
= & \left(b_{s} ; \theta_{s}, \phi_{s} ; \theta_{s}^{\prime}, \phi_{s}^{\prime}\right)_{L R} \oplus\left(m_{s}^{\prime} ; e_{s}^{\prime}, f_{s}^{\prime} ; g_{s}^{\prime}, h_{s}^{\prime}\right)_{L R}, \quad \forall s \in M_{3}, \\
& \sum_{j=1}^{n}\left(a_{r j} ; \rho_{r j}, \tau_{r j} ; \rho_{r j}^{\prime} \tau_{r j}^{\prime}\right)_{L R} \otimes\left(x_{j} ; \alpha_{j}, \beta_{j} ; \alpha_{j}^{\prime}, \beta_{j}^{\prime}\right)_{L R}=\left(b_{r} ; \theta_{r}, \phi_{r} ; \theta_{r}^{\prime}, \phi_{r}^{\prime}\right)_{L R}, \quad \forall r \in M_{2}, \\
& \Re\left(m_{l} ; e_{l}, f_{l} ; g_{l}, h_{l}\right)_{L R}-\Re\left(m_{l}^{\prime} ; e_{l}^{\prime}, f_{l}^{\prime} ; g_{l}^{\prime}, h_{l}^{\prime}\right)_{L R} \geq 0, \quad \forall l \in M_{1}, \\
& \Re\left(m_{s} ; e_{s}, f_{s} ; g_{s}, h_{s}\right)_{L R}-\Re\left(m_{s}^{\prime} ; e_{s}^{\prime}, f_{s}^{\prime} ; g_{s}^{\prime}, h_{s}^{\prime}\right)_{L R} \leq 0, \quad \forall s \in M_{3},
\end{aligned}
$$

where $\left(x_{j} ; \alpha_{j}, \beta_{j} ; \alpha_{j}^{\prime}, \beta_{j}^{\prime}\right)_{L R},\left(m_{l} ; e_{l}, f_{l} ; g_{l}, h_{l}\right)_{L R},\left(m_{l}^{\prime} ; e_{l}^{\prime}\right.$, $\left.f_{l}^{\prime} ; \quad g_{l}^{\prime}, h_{l}^{\prime}\right)_{L R}, \quad\left(m_{s} ; e_{s}, f_{s} ; g_{s}, h_{s}\right)_{L R}, \quad$ and $\left(m_{s}^{\prime} ; e_{s}^{\prime}, f_{s}^{\prime} ;\right.$ $\left.g_{s}^{\prime}, h_{s}^{\prime}\right)_{L R}$ are $L R$-type PFN.

$$
\begin{aligned}
\operatorname{Max} / \operatorname{Min} & \sum_{j=1}^{n}\left(c_{j} ; \zeta_{j}, \eta_{j} ; \zeta_{j}^{\prime}, \eta_{j}^{\prime}\right)_{L R} \otimes\left(x_{j} ; \alpha_{j}, \beta_{j} ; \alpha_{j}^{\prime}, \beta_{j}^{\prime}\right)_{L R}, \\
& \sum_{j=1}^{n}\left(\lambda_{l j} ; \delta_{l j}, \gamma_{l j} ; \delta_{l j}^{\prime}, \gamma_{l j}^{\prime}\right)_{L R} \oplus\left(m_{l} ; e_{l}, f_{l} ; g_{l}, h_{l}\right)_{L R}=\left(b_{l} ; \theta_{l}, \phi_{l} ; \theta_{l}^{\prime}, \phi_{l}^{\prime}\right)_{L R} \oplus\left(m_{l}^{\prime} ; e_{l}^{\prime}, f_{l}^{\prime} ; g_{l}^{\prime}, h_{l}^{\prime}\right)_{L R}, \quad \forall l \in M_{1}, \\
& \sum_{j=1}^{n}\left(\lambda_{s j} ; \delta_{s j}, \gamma_{s j} ; \delta_{s j}^{\prime} \gamma_{s}^{\prime}\right)_{L R} \oplus\left(m_{s} ; e_{s}, f_{s} ; g_{s}, h_{s}\right)_{L R}=\left(b_{s} ; \theta_{s}, \phi_{s} ; \theta_{s}^{\prime}, \phi_{s}^{\prime}\right)_{L R} \oplus\left(m_{s}^{\prime} ; e_{s}^{\prime}, f_{s}^{\prime} ; g_{s}^{\prime}, h_{s}^{\prime}\right)_{L R}, \quad \forall s \in M_{3}, \\
& \sum_{j=1}^{n}\left(\lambda_{r j} ; \delta_{r j}, \gamma_{r j} ; \delta_{r j}^{\prime} \gamma_{r}^{\prime}\right)_{L R}=\left(b_{r} ; \theta_{r}, \phi_{r} ; \theta_{r}^{\prime}, \phi_{r}^{\prime}\right)_{L R}, \quad \forall r \in M_{2}, \\
& \Re\left(m_{l} ; e_{l}, f_{l} ; g_{l}, h_{l}\right)_{L R}-\Re\left(m_{l}^{\prime} ; e_{l}^{\prime}, f_{l}^{\prime} ; g_{l}^{\prime}, h_{l}^{\prime}\right)_{L R} \geq 0, \quad \forall l \in M_{1}, \\
& \Re\left(m_{s} ; e_{s}, f_{s} ; g_{s}, h_{s}\right)_{L R}-\Re\left(m_{s}^{\prime} ; e_{s}^{\prime}, f_{s}^{\prime} ; g_{s}^{\prime}, h_{s}^{\prime}\right)_{L R} \leq 0, \quad \forall s \in M_{3},
\end{aligned}
$$

Step 4: by using the product as discussed in Section 2 and taking $\left(a_{i j} ; \rho_{i j}, \tau_{i j} ; \rho_{i j}^{\prime}, \tau_{i j}^{\prime}\right)_{L R} \otimes\left(x_{j} ; \alpha_{j}, \beta_{j} ; \alpha_{j}^{\prime} \beta_{j}^{\prime}\right)_{L R}=$ $\left(\lambda_{i j} ; \delta_{i j}, \gamma_{i j} ; \delta_{i j}^{\prime}, \gamma_{i j}^{\prime}\right)_{L R}$, the FPFLPP (21) can be written as

where $\left(x_{j} ; \alpha_{j}, \beta_{j} ; \alpha_{j}^{\prime}, \beta_{j}^{\prime}\right)_{L R}, \quad\left(m_{l} ; e_{l}, f_{l} ; g_{l}, h_{l}\right)_{L R},\left(m_{l}^{\prime} ; e_{l}^{\prime}\right.$, $\left.f_{l}^{\prime} ; \quad g_{l}^{\prime}, h_{l}^{\prime}\right)_{L R}, \quad\left(m_{s} ; e_{s}, f_{s} ; g_{s}, h_{s}\right)_{L R}, \quad$ and $\left(m_{s}^{\prime} ; e_{s}^{\prime}, f_{s}^{\prime}\right.$; $\left.g_{s}^{\prime}, h_{s}^{\prime}\right)_{L R}$ are LR-type PFN.

$\operatorname{Max} / \operatorname{Min} \sum_{j=1}^{n}\left(c_{j} ; \zeta_{j}, \eta_{j} ; \zeta_{j}^{\prime}, \eta_{j}^{\prime}\right)_{L R} \otimes\left(x_{j} ; \alpha_{j}, \beta_{j} ; \alpha_{j}^{\prime}, \beta_{j}^{\prime}\right)_{L R^{\prime}}$

Step 5: using arithmetic operations as discussed in Section 2 and using Definition 6, the FPFLPP (23) takes the form

which subject to 


$$
\begin{aligned}
& \sum_{j=1}^{n} \lambda_{l j}+m_{l}=b_{1}+m_{1}^{\prime}, \quad \forall l \in M_{1}, \\
& \sum_{j=1}^{n} \delta_{l j}+e_{l}=\theta_{l}+e_{l}^{\prime}, \quad \forall l \in M_{1}, \\
& \sum_{j=1}^{n} \gamma_{l j}+f_{l}=\phi_{l}+f_{l}^{\prime}, \quad \forall l \in M_{1}, \\
& \sum_{j=1}^{n} \delta_{l j}^{\prime}+g_{l}^{\prime}=\theta_{l}^{\prime}+g_{l}^{\prime}, \quad \forall l \in M_{1}, \\
& \sum_{j=1}^{n} \gamma_{l j}^{\prime}+h_{l}=\phi_{l}^{\prime}+h_{l}^{\prime}, \quad \forall l \in M_{1}, \\
& \sum_{j=1}^{n} \lambda_{r j}=b_{r}, \quad \forall r \in M_{2}, \\
& \sum_{j=1}^{n} \gamma_{r j}=\theta_{r}^{\prime}, \quad \forall r \in M_{2}, \\
& \sum_{j=1}^{n} \delta_{r j}^{n} \gamma_{s j}^{\prime}+h_{s}=\theta_{r}^{\prime}, \quad \forall r \in h_{s}^{\prime}, \quad \forall s \in M_{3}, \\
& \sum_{j=1}^{n} \delta_{s j}^{\prime} \delta_{s j}+g_{s}^{\prime}=\theta_{s}^{\prime}+g_{s}^{\prime}, \quad \forall s \in M_{3}, \\
& \sum_{j=1}^{n} \gamma_{r j}^{\prime}=\phi_{r}^{\prime}, \quad \forall r \in M_{2},
\end{aligned}
$$$$
\mathfrak{R}\left(m_{l} ; e_{l}, f_{l} ; g_{l}, h_{l}\right)_{L R}
$$$$
-\mathfrak{R}\left(m_{l}^{\prime} ; e_{l}^{\prime}, f_{l}^{\prime} ; g_{l}^{\prime}, h_{l}^{\prime}\right)_{L R} \geq 0, \quad \forall l \in M_{1} \text {, }
$$$$
\Re\left(m_{s} ; e_{s}, f_{s} ; g_{s}, h_{s}\right)_{L R}
$$$$
-\mathfrak{R}\left(m_{s}^{\prime} ; e_{s}^{\prime}, f_{s}^{\prime} ; g_{s}^{\prime}, h_{s}^{\prime}\right)_{L R} \leq 0, \quad \forall s \in M_{3} \text {, }
$$

where $\left(x_{j} ; \alpha_{j}, \beta_{j} ; \alpha_{j}^{\prime}, \beta_{j}^{\prime}\right)_{L R}, \quad\left(m_{l} ; e_{l}, f_{l} ; g_{l}, h_{l}\right)_{L R}, \quad\left(m_{l}^{\prime} ; e_{l}^{\prime}\right.$, $\left.f_{l}^{\prime} ; g_{l}^{\prime}, h_{l}^{\prime}\right)_{L R}, \quad\left(m_{s} ; e_{s}, f_{s} ; g_{s}, h_{s}\right)_{L R}$, and $\left(m_{s}^{\prime} ; e_{s}^{\prime}, f_{s}^{\prime} ; g_{s}^{\prime}\right.$, $\left.h_{s}^{\prime}\right)_{L R}$ are $L R$-type PFN.

Step 6: now, we have to find $L R$-type Pythagorean fuzzy feasible solution out of all $L R$-type Pythagorean fuzzy feasible solutions corresponding to which the ranking of the objective is optimum. By applying ranking, the FPFLPP (24) can be written as

$\operatorname{Max} / \operatorname{Min} \Re\left(\sum_{j=1}^{n}\left(c_{j} ; \zeta_{j}, \eta_{j} ; \zeta_{j}^{\prime}, \eta_{j}^{\prime}\right)_{L R} \otimes\left(x_{j} ; \alpha_{j}, \beta_{j} ; \alpha_{j}^{\prime}, \beta_{j}^{\prime}\right)_{L R}\right)$,

which subject to

$$
\begin{aligned}
& \sum_{j=1}^{n} \lambda_{l j}+m_{l}=b_{1}+m_{1}^{\prime}, \quad \forall l \in M_{1}, \\
& \sum_{j=1}^{n} \delta_{l j}+e_{l}=\theta_{l}+e_{l}^{\prime}, \quad \forall l \in M_{1}, \\
& \sum_{j=1}^{n} \gamma_{l j}+f_{l}=\phi_{l}+f_{l}^{\prime}, \quad \forall l \in M_{1}, \\
& \sum_{j=1}^{n} \delta_{l j}^{\prime}+g_{l}^{\prime}=\theta_{l}^{\prime}+g_{l}^{\prime}, \quad \forall l \in M_{1}, \\
& \sum_{j=1}^{n} \gamma_{l j}^{\prime}+h_{l}=\phi_{l}^{\prime}+h_{l}^{\prime}, \quad \forall l \in M_{1}, \\
& \sum_{j=1}^{n} \lambda_{r j}=b_{r}, \quad \forall r \in M_{2}, \\
& \sum_{j=1}^{n} \delta_{r j}=\theta_{r}, \quad \forall r \in M_{2}, \\
& \sum_{j=1}^{n} \gamma_{r j}=\phi_{r}, \quad \forall r \in M_{2}, \\
& \sum_{j=1}^{n} \gamma_{r j}^{\prime}=\phi_{r}^{\prime}, \quad \forall r \in M_{2}^{\prime}, \quad \forall r \in M_{2}, \\
& \sum_{j=1}^{n} \lambda_{s j}^{n} \gamma_{s j}^{n} \delta_{s j}^{\prime}+f_{s}, \quad \forall s=\phi_{s}^{\prime}+h_{s}^{\prime}, \quad \forall s \in M_{3}, \\
& \sum_{s}^{n}=\theta_{s}, \quad \forall s \in M_{3},
\end{aligned}
$$

$\Re\left(m_{l} ; e_{l}, f_{l} ; g_{l}, h_{l}\right)_{L R}$

$-\Re\left(m_{l}^{\prime} ; e_{l}^{\prime}, f_{l}^{\prime} ; g_{l}^{\prime}, h_{l}^{\prime}\right)_{L R} \geq 0, \quad \forall l \in M_{1}$,

$\mathfrak{R}\left(m_{s} ; e_{s}, f_{s} ; g_{s}, h_{s}\right)_{L R}$

$-\Re\left(m_{s}^{\prime} ; e_{s}^{\prime}, f_{s}^{\prime} ; g_{s}^{\prime}, h_{s}^{\prime}\right)_{L R} \leq 0, \quad \forall s \in M_{3}$, 
$\alpha_{j} \geq 0, \beta_{j} \geq 0, \alpha_{j}^{\prime}-\alpha_{j} \geq 0, \beta_{j}^{\prime}-\beta_{j} \geq 0, e_{i} \geq 0, f_{i} \geq 0, e_{i}^{\prime}$ $-e_{i} \geq 0, \quad$ and $f_{i}^{\prime}-f_{i} \geq 0, \quad \forall i=1,2, \ldots, m$ and $\forall j=1,2, \ldots, n$.

Step 7: by taking $\left(c_{j} ; \zeta_{j}, \eta_{j} ; \zeta_{j}^{\prime}, \eta_{j}^{\prime}\right)_{L R} \otimes\left(x_{j} ; \alpha_{j}\right.$, $\left.\beta_{j} ; \alpha_{j}^{\prime}, \beta_{j}^{\prime}\right)_{L R}=\left(\omega_{j} ; \sigma_{j}, \kappa_{j} ; \sigma_{j}^{\prime}, \kappa_{j}^{\prime}\right)_{L R}$, problem (26) can be written as

$$
\operatorname{Max} / \operatorname{Min} \Re\left(\sum_{j=1}^{n}\left(\omega_{j} ; \sigma_{j}, \kappa_{j} ; \sigma_{j}^{\prime}, \kappa_{j}^{\prime}\right)_{L R}\right),
$$

which subject to

$$
\begin{aligned}
& \sum_{j=1}^{n} \lambda_{l j}+m_{l}=b_{1}+m_{1}^{\prime}, \quad \forall l \in M_{1}, \\
& \sum_{j=1}^{n} \delta_{l j}+e_{l}=\theta_{l}+e_{l}^{\prime}, \quad \forall l \in M_{1}, \\
& \sum_{j=1}^{n} \gamma_{l j}+f_{l}=\phi_{l}+f_{l}^{\prime}, \quad \forall l \in M_{1}, \\
& \sum_{j=1}^{n} \delta_{l j}^{\prime}+g_{l}^{\prime}=\theta_{l}^{\prime}+g_{l}^{\prime}, \quad \forall l \in M_{1}, \\
& \sum_{j=1}^{n} \gamma_{l j}^{\prime}+h_{l}=\phi_{l}^{\prime}+h_{l}^{\prime}, \quad \forall l \in M_{1}, \\
& \sum_{j=1}^{n} \lambda_{r j}=b_{r}, \quad \forall r \in M_{2}, \\
& \sum_{j=1}^{n} \delta_{r j}=\theta_{r}, \quad \forall r \in M_{2}, \\
& \sum_{j=1}^{n} \gamma_{r j}^{\prime}=\phi_{r}^{\prime}, \quad \forall r \in M_{2}, \\
& \sum_{j=1}^{n} \lambda_{s j}+m_{s}=b_{s}+m_{s}^{\prime}, \quad \forall s \in M_{3}, \\
& \sum_{j=1}^{n} \delta_{s j}+e_{s}=\theta_{s}+e_{s}^{\prime}, \quad \forall s \in M_{3}, \\
& \sum_{j=1}^{n} \gamma_{s j}+f_{s}=\phi_{s}+f_{s}^{\prime}, \quad \forall s \in M_{3}, \\
& \sum_{j=1}^{n} \delta_{s j}^{\prime}+g_{s}^{\prime}=\theta_{s}^{\prime}+g_{s}^{\prime}, \quad \forall s \in M_{3}, \\
& \sum_{j=1}^{n} \gamma_{r j}=\phi_{r}, \quad \forall r \in M_{s}^{\prime}+h_{s}^{\prime}, \quad \forall s \in M_{3}, \\
& \sum_{j=1}^{n} \delta_{r j}^{\prime}=\theta_{r}^{\prime}, \quad \forall r \in M_{2},
\end{aligned}
$$$$
\mathfrak{R}\left(m_{l} ; e_{l}, f_{l} ; g_{l}, h_{l}\right)_{L R}
$$$$
-\Re\left(m_{l}^{\prime} ; e_{l}^{\prime}, f_{l}^{\prime} ; g_{l}^{\prime}, h_{l}^{\prime}\right)_{L R} \geq 0, \quad \forall l \in M_{1},
$$$$
\Re\left(m_{s} ; e_{s}, f_{s} ; g_{s}, h_{s}\right)_{L R}
$$$$
-\Re\left(m_{s}^{\prime} ; e_{s}^{\prime}, f_{s}^{\prime} ; g_{s}^{\prime}, h_{s}^{\prime}\right)_{L R} \leq 0, \quad \forall s \in M_{3} \text {, }
$$

$\alpha_{j} \geq 0, \beta_{j} \geq 0, \alpha_{j}^{\prime}-\alpha_{j} \geq 0, \beta_{j}^{\prime}-\beta_{j} \geq 0, e_{i} \geq 0, f_{i} \geq 0, e_{i}^{\prime}$ $-e_{i} \geq 0, \quad$ and $f_{i}^{\prime}-f_{i} \geq 0, \quad \forall i=1,2, \ldots, m \quad$ and $\forall j=1,2, \ldots, n$.

Step 8: by using the linearity property of ranking function, problem (28) takes the form

$$
\operatorname{Max} / \operatorname{Min}\left(\sum_{j=1}^{n} \mathfrak{R}\left(\omega_{j} ; \sigma_{j}, \kappa_{j} ; \sigma_{j}^{\prime}, \kappa_{j}^{\prime}\right)_{L R}\right),
$$

which subject to

$$
\begin{aligned}
& \sum_{j=1}^{n} \lambda_{l j}+m_{l}=b_{1}+m_{1}^{\prime}, \quad \forall l \in M_{1}, \\
& \sum_{j=1}^{n} \delta_{l j}+e_{l}=\theta_{l}+e_{l}^{\prime}, \quad \forall l \in M_{1}, \\
& \sum_{j=1}^{n} \gamma_{l j}+f_{l}=\phi_{l}+f_{l}^{\prime}, \quad \forall l \in M_{1}, \\
& \sum_{j=1}^{n} \delta_{l j}^{\prime}+g_{l}^{\prime}=\theta_{l}^{\prime}+g_{l}^{\prime}, \quad \forall l \in M_{1}, \\
& \sum_{j=1}^{n} \gamma_{l j}^{\prime}+h_{l}=\phi_{l}^{\prime}+h_{l}^{\prime}, \quad \forall l \in M_{1}, \\
& \sum_{j=1}^{n} \lambda_{r j}=b_{r}, \quad \forall r \in M_{2}, \\
& \sum_{j=1}^{n} \delta_{r j}=\theta_{r}, \quad \forall r \in M_{2}, \\
& \sum_{j=1}^{n} \gamma_{r j}^{\prime}=\phi_{r}^{\prime}, \quad \forall r \in M_{2}, \\
& \sum_{j=1}^{n} \lambda_{s j}+m_{s}=b_{s}+m_{s}^{\prime}, \quad \forall s \in M_{3}, \\
& \sum_{j=1}^{n} \delta_{s j}+e_{s}=\theta_{s}+e_{s}^{\prime}, \quad \forall s \in M_{3}, \\
& \sum_{j=1}^{n} \gamma_{s j}+f_{s}=\phi_{s}+f_{s}^{\prime}, \quad \forall s \in M_{3}, \\
& \sum_{j=1}^{n} \delta_{s j}^{\prime}+g_{s}^{\prime}=\theta_{s}^{\prime}+g_{s}^{\prime}, \quad \forall s \in M_{3}, \\
& \sum_{j=1}^{n} \gamma_{r j}=\phi_{r}, \quad \forall r \in h_{s}=\phi_{s}^{\prime}+h_{s}^{\prime}, \quad \forall s \in M_{3},
\end{aligned}
$$

$$
\begin{aligned}
& \Re\left(m_{l} ; e_{l}, f_{l} ; g_{l}, h_{l}\right)_{L R} \\
& -\mathfrak{R}\left(m_{l}^{\prime} ; e_{l}^{\prime}, f_{l}^{\prime} ; g_{l}^{\prime}, h_{l}^{\prime}\right)_{L R} \geq 0, \quad \forall l \in M_{1}, \\
& \mathfrak{R}\left(m_{s} ; e_{s}, f_{s} ; g_{s}, h_{s}\right)_{L R} \\
& -\mathfrak{R}\left(m_{s}^{\prime} ; e_{s}^{\prime}, f_{s}^{\prime} ; g_{s}^{\prime}, h_{s}^{\prime}\right)_{L R} \leq 0, \quad \forall s \in M_{3},
\end{aligned}
$$


$\alpha_{j} \geq 0, \beta_{j} \geq 0, \alpha_{j}^{\prime}-\alpha_{j} \geq 0, \beta_{j}^{\prime}-\beta_{j} \geq 0, e_{i} \geq 0, f_{i} \geq 0, e_{i}^{\prime}$ $-e_{i} \geq 0$, and $f_{i}^{\prime}-f_{i} \geq 0, \quad \forall i=1,2, \ldots, m \quad$ and $\forall j=1,2, \ldots, n$.
Step 9: by using Definition 7, problem (30) can be converted into

$\operatorname{Max} / \operatorname{Min} \sum_{j=1}^{n}\left[\frac{1}{4}\left\{\left(\int_{0}^{1}\left(\omega_{j}-\sigma_{j} L^{-1}(\alpha)\right) \mathrm{d} \alpha+\int_{0}^{1}\left(\omega_{j}+\kappa_{j} R^{-1}(\alpha)\right) \mathrm{d} \alpha\right)+\left(\int_{0}^{1}\left(\omega_{j}-\sigma_{j}^{\prime} L^{\prime-1}(\alpha)\right) \mathrm{d} \alpha+\int_{0}^{1}\left(\omega_{j}+\kappa_{j}^{\prime} R^{\prime-1}(\alpha)\right) \mathrm{d} \alpha\right)\right\}\right]$

which subject to

$$
\begin{aligned}
& \sum_{j=1}^{n} \lambda_{l j}+m_{l}=b_{1}+m_{1}^{\prime}, \quad \forall l \in M_{1}, \\
& \sum_{j=1}^{n} \delta_{l j}+e_{l}=\theta_{l}+e_{l}^{\prime}, \quad \forall l \in M_{1}, \\
& \sum_{j=1}^{n} \gamma_{l j}+f_{l}=\phi_{l}+f_{l}^{\prime}, \quad \forall l \in M_{1}, \\
& \sum_{j=1}^{n} \delta_{l j}^{\prime}+g_{l}^{\prime}=\theta_{l}^{\prime}+g_{l}^{\prime}, \quad \forall l \in M_{1}, \\
& \sum_{j=1}^{n} \gamma_{l j}^{\prime}+h_{l}=\phi_{l}^{\prime}+h_{l}^{\prime}, \quad \forall l \in M_{1}, \\
& \sum_{j=1}^{n} \gamma_{s j}+f_{s}=\phi_{s}+f_{s}^{\prime}, \quad \forall s \in M_{3}, \\
& \sum_{j=1}^{n} \lambda_{j=1}^{n} \lambda_{r j}=b_{r}, \quad \forall r \in g_{s}^{\prime}, \quad \forall s \in M_{3}, \\
& \sum_{j=1}^{n} \gamma_{r j}^{\prime} \delta_{r j}=\phi_{r}^{\prime}, \quad \forall r \in M_{2}, \quad \forall r \in M_{2},
\end{aligned}
$$




$$
\begin{aligned}
& \sum_{j=1}^{n} \gamma_{s j}^{\prime}+h_{s}=\phi_{s}^{\prime}+h_{s}^{\prime}, \quad \forall s \in M_{3}, \\
& \sum_{j=1}^{n} \gamma_{r j}=\phi_{r}, \quad \forall r \in M_{2}, \\
& \sum_{j=1}^{n} \delta_{r j}^{\prime}=\theta_{r}^{\prime}, \quad \forall r \in M_{2}, \\
& \quad \frac{1}{4}\left\{\left(\int_{0}^{1}\left(m_{l}-e_{l} L^{-1}(\alpha)\right) \mathrm{d} \alpha+\int_{0}^{1}\left(m_{l}+f_{l} R^{-1}(\alpha)\right) \mathrm{d} \alpha\right)+\left(\int_{0}^{1}\left(m_{l}-g_{l} L^{\prime-1}(\alpha)\right) \mathrm{d} \alpha+\int_{0}^{1}\left(m_{l}+h_{l} R^{\prime-1}(\alpha)\right) \mathrm{d} \alpha\right)\right\} \\
& \quad-\frac{1}{4}\left\{\left(\int_{0}^{1}\left(m_{l}^{\prime}-e_{l}^{\prime} L^{-1}(\alpha)\right) \mathrm{d} \alpha+\int_{0}^{1}\left(m_{l}^{\prime}+f_{l}^{\prime} R^{-1}(\alpha)\right) \mathrm{d} \alpha\right)+\left(\int_{0}^{1}\left(m_{l}^{\prime}-g_{l}^{\prime} L^{-1}(\alpha)\right) \mathrm{d} \alpha+\int_{0}^{1}\left(m_{l}^{\prime}+h_{l}^{\prime} R^{\prime-1}(\alpha)\right) \mathrm{d} \alpha\right)\right\} \\
& \quad \geq 0, \quad \forall l \in M_{1}, \\
& \frac{1}{4}\left\{\left(\int_{0}^{1}\left(m_{s}-e_{s} L^{-1}(\alpha)\right) \mathrm{d} \alpha+\int_{0}^{1}\left(m_{s}+f_{s} R^{-1}(\alpha)\right) \mathrm{d} \alpha\right)+\left(\int_{0}^{1}\left(m_{s}-g_{s} L^{-1}(\alpha)\right) \mathrm{d} \alpha+\int_{0}^{1}\left(m_{s}+h_{s} R^{\prime-1}(\alpha)\right) \mathrm{d} \alpha\right)\right\} \\
& \quad-\frac{1}{4}\left\{\left(\int_{0}^{1}\left(m_{s}^{\prime}-e_{s}^{\prime} L^{-1}(\alpha)\right) \mathrm{d} \alpha+\int_{0}^{1}\left(m_{s}^{\prime}+f_{s}^{\prime} R^{-1}(\alpha)\right) \mathrm{d} \alpha\right)+\left(\int_{0}^{1}\left(m_{s}^{\prime}-g_{s}^{\prime} L^{\prime-1}(\alpha)\right) \mathrm{d} \alpha+\int_{0}^{1}\left(m_{s}^{\prime}+h_{s}^{\prime} R^{-1}(\alpha)\right) \mathrm{d} \alpha\right)\right\} \\
& \quad \leq 0, \quad \forall l \in M_{3},
\end{aligned}
$$

$\alpha_{j} \geq 0, \beta_{j} \geq 0, \alpha_{j}^{\prime}-\alpha_{j} \geq 0, \beta_{j}^{\prime}-\beta_{j} \geq 0, e_{i} \geq 0, f_{i} \geq 0, e_{i}^{\prime}$ $-e_{i} \geq 0, \quad$ and $f_{i}^{\prime}-f_{i} \geq 0, \quad \forall i=1,2, \ldots, m$ and $\forall j=1,2, \ldots, n$.

Step 10: now, solve the crisp linear/nonlinear programming problem (32) by any existing method to find the optimal solution $x_{j}^{*}, \alpha_{j}^{*}, \beta_{j}^{*}, \alpha_{j}^{*}, \beta_{j}^{*}$.

Step 11: find the $L R$-type Pythagorean fuzzy optimal solution $X_{j}^{*}$ of the FPFLPP (13) by substituting the values of $x_{j}^{*}, \alpha_{j}^{*}, \beta_{j}^{*}, \alpha_{j}^{\prime *}, \quad$ and $\beta_{j}^{*}$ in $X_{j}^{*}=\left(x_{j}^{*} ; \alpha_{j}^{*}, \beta_{j}^{*} ; \alpha_{j}^{\prime *}, \beta_{j}^{\prime *}\right)_{L R}$.

Step 12: find the $L R$-type Pythagorean fuzzy optimal value of the FPFLPP (13) by substituting the values of $X_{j}^{*}$, as calculated in Step (11), in $\sum_{j=1}^{n} C_{j} \otimes X_{j}$.

3.2. Method 2: FPFLPP Using Ranking Function. Now, we present another method to solve FPFLPP (13). We present a criterion for the optimal solution.

Definition 9. An LR-type Pythagorean fuzzy optimal solution of FPFLPP (13) with LR-type PFNs will be $L R$-type PFNs $X_{j}$ if
(1) $X_{j}$ are $L R$-type PFNs

(2) $\mathfrak{R}\left(\sum_{j=1}^{n} A_{i j} \otimes X_{j}\right) \leq,=, \geq \mathfrak{R}\left(B_{i}\right)$, for all $i=1,2$, $\ldots, m$

(3) If there exist any $L R$-type PFNs $X_{j}^{\prime}$ satisfying step 2, then $\mathfrak{R}\left(\sum_{j=1}^{n} C_{j} \otimes X_{j}\right)>\mathfrak{R}\left(\sum_{j=1}^{n} C_{j} \otimes X_{j}^{\prime}\right)$ in maximization problem and $\mathfrak{R}\left(\sum_{j=1}^{n} C_{j} \otimes X_{j}\right)<\mathfrak{R}$ $\left(\sum_{j=1}^{n} C_{j} \otimes X_{j}^{\prime}\right)$ in minimization problem

Step 1: by assuming $A_{i j}=\left(a_{i j} ; \rho_{i j}, \tau_{i j} ; \rho_{i j}{ }^{\prime}, \tau_{i j}^{\prime}\right)_{L R}$, $X_{j}=\left(x_{j} ; \alpha_{j}, \beta_{j} ; \alpha_{j}^{\prime}, \beta_{j}^{\prime}\right)_{L R}, \quad B_{i}=\left(b_{i} ; \theta_{i}, \phi_{i} ; \theta_{i}^{\prime}, \phi_{i}^{\prime}\right)_{L R}$, and $C_{j}=\left(c_{j} ; \zeta_{j}, \eta_{j} ; \zeta_{j}^{\prime}, \eta_{j}^{\prime}\right)_{L R}$, the FPFLPP (15) can be rewritten as

$$
\operatorname{Max} / \operatorname{Min} \sum_{j=1}^{n}\left(c_{j} ; \zeta_{j}, \eta_{j} ; \zeta_{j}^{\prime}, \eta_{j}^{\prime}\right)_{L R} \otimes\left(x_{j} ; \alpha_{j}, \beta_{j} ; \alpha_{j}^{\prime}, \beta_{j}^{\prime}\right)_{L R^{\prime}}
$$

which subject to

$$
\begin{array}{ll}
\sum_{j=1}^{n}\left(a_{l j} ; \rho_{l j}, \tau_{l j} ; \rho_{l j}^{\prime}, \tau_{l j}^{\prime}\right)_{L R} \otimes\left(x_{j} ; \alpha_{j}, \beta_{j} ; \alpha_{j}^{\prime}, \beta_{j}^{\prime}\right)_{L R} \preccurlyeq\left(b_{l} ; \theta_{l}, \phi_{l} ; \theta_{l}^{\prime}, \phi_{l}^{\prime}\right)_{L R}, \quad \forall l \in M_{1}, \\
\sum_{j=1}^{n}\left(a_{s j} ; \rho_{s j}, \tau_{s j} ; \rho_{s j}^{\prime} \tau_{s}^{\prime}\right)_{L R} \otimes\left(x_{j} ; \alpha_{j}, \beta_{j} ; \alpha_{j}^{\prime}, \beta_{j}^{\prime}\right)_{L R} \pm\left(b_{s} ; \theta_{s}, \phi_{s} ; \theta_{s}^{\prime}, \phi_{s}^{\prime}\right)_{L R}, \quad \forall s \in M_{3}, \\
\sum_{j=1}^{n}\left(a_{r j} ; \rho_{r j}, \tau_{r j} ; \rho_{r j}^{\prime} \tau_{r}^{\prime}\right)_{L R} \otimes\left(x_{j} ; \alpha_{j}, \beta_{j} ; \alpha_{j}^{\prime}, \beta_{j}^{\prime}\right)_{L R}=\left(b_{r} ; \theta_{r}, \phi_{r} ; \theta_{r}^{\prime}, \phi_{r}^{\prime}\right)_{L R}, \quad \forall r \in M_{2},
\end{array}
$$


where $\left(x_{j} ; \alpha_{j}, \beta_{j} ; \alpha_{j}^{\prime}, \beta_{j}^{\prime}\right)_{L R}$ is an $L R$-type PFN.

Step 2: by using the product as discussed in Section 2 and taking $\left(a_{i j} ; \rho_{i j}, \tau_{i j} ; \rho_{i j}^{\prime}, \tau_{i j}^{\prime}\right)_{L R} \otimes\left(x_{j} ;\right.$ $\left.\alpha_{j}, \beta_{j} \alpha_{j}^{\prime}, \beta_{j}^{\prime}\right)_{L R}=\left(\lambda_{i j} ; \delta_{i j}, \gamma_{i j} ; \delta_{i j}^{\prime}, \gamma_{i j}^{\prime}\right)_{L R}$, the FPFLPP (34) can be written as

$$
\begin{aligned}
\operatorname{Max} / \operatorname{Min} & \sum_{j=1}^{n}\left(c_{j} ; \zeta_{j}, \eta_{j} ; \zeta_{j}^{\prime}, \eta_{j}^{\prime}\right)_{L R} \otimes\left(x_{j} ; \alpha_{j}, \beta_{j} ; \alpha_{j}^{\prime}, \beta_{j}^{\prime}\right)_{L R}, \\
& \sum_{j=1}^{n}\left(\lambda_{l j} ; \delta_{l j}, \gamma_{l j} ; \delta_{l j}^{\prime}, \gamma_{l j}^{\prime}\right)_{L R} \preccurlyeq\left(b_{l} ; \theta_{l}, \phi_{l} ; \theta_{l}^{\prime}, \phi_{l}^{\prime}\right)_{L R}, \quad \forall l \in M_{1}, \\
& \sum_{j=1}^{n}\left(\lambda_{s j} ; \delta_{s j}, \gamma_{s j} ; \delta_{s j}^{\prime} \gamma_{s}^{\prime}\right)_{L R} \geqslant\left(b_{s} ; \theta_{s}, \phi_{s} ; \theta_{s}^{\prime}, \phi_{s}^{\prime}\right)_{L R}, \quad \forall s \in M_{3}, \\
& \sum_{j=1}^{n}\left(\lambda_{r j} ; \delta_{r j}, \gamma_{r j} ; \delta_{r j}^{\prime} \gamma_{r j}^{\prime}\right)_{L R}=\left(b_{r} ; \theta_{r}, \phi_{r} ; \theta_{r}^{\prime}, \phi_{r}^{\prime}\right)_{L R}, \quad \forall r \in M_{2},
\end{aligned}
$$

where $\left(x_{j} ; \alpha_{j}, \beta_{j} ; \alpha_{j}^{\prime}, \beta_{j}^{\prime}\right)_{L R}$ is an $L R$-type PFN.

Step 3: using arithmetic operations as discussed in

$$
\operatorname{Max} / \operatorname{Min} \sum_{j=1}^{n}\left(c_{j} ; \zeta_{j}, \eta_{j} ; \zeta_{j}^{\prime}, \eta_{j}^{\prime}\right)_{L R} \otimes\left(x_{j} ; \alpha_{j}, \beta_{j} ; \alpha_{j}^{\prime}, \beta_{j}^{\prime}\right)_{L R}
$$

Section 2 and using Definition 6, the FPFLPP (36)

can be rewritten as

which subject to

$$
\begin{aligned}
& \sum_{j=1}^{n}\left(\lambda_{l j} ; \delta_{l j}, \gamma_{l j} ; \delta_{l j}^{\prime}, \gamma_{l j}^{\prime}\right)_{L R} \leqslant\left(b_{l} ; \theta_{l}, \phi_{l} ; \theta_{l}, \phi_{l}^{\prime}\right)_{L R}, \quad \forall l \in M_{1}, \\
& \sum_{j=1}^{n}\left(\lambda_{s j} ; \delta_{s j}, \gamma_{s j} ; \delta_{s j}^{\prime} \gamma_{s}^{\prime}\right)_{L R} \geqslant\left(b_{s} ; \theta_{s}, \phi_{s} ; \theta_{s}^{\prime}, \phi_{s}^{\prime}\right)_{L R}, \quad \forall s \in M_{3}, \\
& \sum_{j=1}^{n} \lambda_{r j}=b_{r}, \quad \forall r \in M_{2}, \\
& \sum_{j=1}^{n} \delta_{r j}=\theta_{r}, \quad \forall r \in M_{2}, \\
& \sum_{j=1}^{n} \gamma_{r j}^{\prime}=\phi_{r}^{\prime}, \quad \forall r \in M_{2}, \\
& \sum_{j=1}^{n} \gamma_{r j}=\phi_{r}, \quad \forall r \in M_{2}, \\
& \sum_{j=1}^{n} \delta_{r j}^{\prime}=\theta_{r}^{\prime}, \quad \forall r \in M_{2},
\end{aligned}
$$

where $\left(x_{j} ; \alpha_{j}, \beta_{j} ; \alpha_{j}^{\prime}, \beta_{j}^{\prime}\right)_{L R}$ is an $L R$-type PFN.

Step 4: by applying ranking, the FPFLPP (37) takes the form

$$
\operatorname{Max} / \operatorname{Min} \Re\left(\sum_{j=1}^{n}\left(c_{j} ; \zeta_{j}, \eta_{j} ; \zeta_{j}^{\prime}, \eta_{j}^{\prime}\right)_{L R} \otimes\left(x_{j} ; \alpha_{j}, \beta_{j} ; \alpha_{j}^{\prime}, \beta_{j}^{\prime}\right)_{L R}\right),
$$


which subject to

$$
\begin{gathered}
\mathfrak{R}\left(\sum_{j=1}^{n}\left(\lambda_{l j} ; \delta_{l j}, \gamma_{l j} ; \delta_{l j}^{\prime}, \gamma_{l j}^{\prime}\right)_{L R}\right) \leq \Re\left(b_{l} ; \theta_{l}, \phi_{l} ; \theta_{l}^{\prime}, \phi_{l}^{\prime}\right)_{L R}, \quad \forall l \in M_{1}, \\
\Re\left(\sum_{j=1}^{n}\left(\lambda_{s j} ; \delta_{s j}, \gamma_{s j} ; \delta_{s j}^{\prime} \gamma_{s}^{\prime}\right)_{L R}\right) \geq \Re\left(b_{s} ; \theta_{s}, \phi_{s} ; \theta_{s}^{\prime}, \phi_{s}^{\prime}\right)_{L R}, \quad \forall s \in M_{3}, \\
\sum_{j=1}^{n} \lambda_{r j}=b_{r}, \quad \forall r \in M_{2}, \\
\sum_{j=1}^{n} \delta_{r j}=\theta_{r}, \quad \forall r \in M_{2}, \\
\sum_{j=1}^{n} \gamma_{r j}^{\prime}=\phi_{r}^{\prime}, \quad \forall r \in M_{2}, \\
\sum_{j=1}^{n} \gamma_{r j}=\phi_{r}, \quad \forall r \in M_{2}, \\
\sum_{j=1}^{n} \delta_{r j}^{\prime}=\theta_{r}^{\prime}, \quad \forall r \in M_{2},
\end{gathered}
$$

$\alpha_{j} \geq 0, \quad \beta_{j} \geq 0, \quad \alpha_{j}^{\prime}-\alpha_{j} \geq 0, \quad$ and $\quad \beta_{j}^{\prime}-\beta_{j} \geq 0$,

$\forall j=1,2, \ldots, n$.

$$
\operatorname{Max} / \operatorname{Min} \Re\left(\sum_{j=1}^{n}\left(\omega_{j} ; \sigma_{j}, \kappa_{j} ; \sigma_{j}^{\prime}, \kappa_{j}^{\prime}\right)_{L R}\right),
$$

Step 5: by taking $\left(c_{j} ; \zeta_{j}, \eta_{j} ; \zeta_{j}^{\prime}, \eta_{j}^{\prime}\right)_{L R} \otimes\left(x_{j} ; \alpha_{j}, \beta_{j} ; \alpha_{j}^{\prime}\right.$, $\left.\beta_{j}^{\prime}\right)_{L R}=\left(\omega_{j} ; \sigma_{j}, \kappa_{j} ; \sigma_{j}^{\prime}, \kappa_{j}^{\prime}\right)_{L R}$, problem (39) can be which subject to rewritten as

$$
\begin{gathered}
\Re\left(\sum_{j=1}^{n}\left(\lambda_{l j} ; \delta_{l j}, \gamma_{l j} ; \delta_{l j}^{\prime}, \gamma_{l j}^{\prime}\right)_{L R}\right) \leq \Re\left(b_{l} ; \theta_{l}, \phi_{l} ; \theta_{l}^{\prime}, \phi_{l}^{\prime}\right)_{L R}, \quad \forall l \in M_{1}, \\
\Re\left(\sum_{j=1}^{n}\left(\lambda_{s j} ; \delta_{s j}, \gamma_{s j} ; \delta_{s j}^{\prime}, \gamma_{s j}^{\prime}\right)_{L R}\right) \geq \Re\left(b_{s} ; \theta_{s}, \phi_{s} ; \theta_{s}^{\prime}, \phi_{s}^{\prime}\right)_{L R}, \quad \forall s \in M_{3}, \\
\sum_{j=1}^{n} \lambda_{r j}=b_{r}, \quad \forall r \in M_{2}, \\
\sum_{j=1}^{n} \delta_{r j}=\theta_{r}, \quad \forall r \in M_{2}, \\
\sum_{j=1}^{n} \gamma_{r j}^{\prime}=\phi_{r}^{\prime}, \quad \forall r \in M_{2}, \\
\sum_{j=1}^{n} \gamma_{r j}=\phi_{r}, \quad \forall r \in M_{2}, \\
\sum_{j=1}^{n} \delta_{r j}^{\prime}=\theta_{r}^{\prime}, \quad \forall r \in M_{2},
\end{gathered}
$$


$\alpha_{j} \geq 0, \quad \beta_{j} \geq 0, \quad \alpha_{j}^{\prime}-\alpha_{j} \geq 0, \quad$ and $\quad \beta_{j}^{\prime}-\beta_{j} \geq 0$, $\forall j=1,2, \ldots, n$.

$$
\operatorname{Max} / \operatorname{Min}\left(\sum_{j=1}^{n} \mathfrak{R}\left(\omega_{j} ; \sigma_{j}, \kappa_{j} ; \sigma_{j}^{\prime}, \kappa_{j}^{\prime}\right)_{L R}\right),
$$

Step 6: by using the linearity of ranking function, problem (41) can be written as which subject to

$$
\begin{gathered}
\left(\sum_{j=1}^{n} \Re\left(\lambda_{l j} ; \delta_{l j}, \gamma_{l j} ; \delta_{l j}^{\prime}, \gamma_{l j}^{\prime}\right)_{L R}\right) \leq \Re\left(b_{l} ; \theta_{l}, \phi_{l} ; \theta_{l}^{\prime}, \phi_{l}^{\prime}\right)_{L R}, \quad \forall l \in M_{1}, \\
\left(\sum_{j=1}^{n} \Re\left(\lambda_{s j} ; \delta_{s j}, \gamma_{s j} ; \delta_{s j}^{\prime} \gamma_{s}^{\prime}\right)_{L R}\right) \geq \Re\left(b_{s} ; \theta_{s}, \phi_{s} ; \theta_{s}^{\prime}, \phi_{s}^{\prime}\right)_{L R}, \quad \forall s \in M_{3}, \\
\sum_{j=1}^{n} \lambda_{r j}=b_{r}, \quad \forall r \in M_{2}, \\
\sum_{j=1}^{n} \delta_{r j}=\theta_{r}, \quad \forall r \in M_{2}, \\
\sum_{j=1}^{n} \gamma_{r j}^{\prime}=\phi_{r}^{\prime}, \quad \forall r \in M_{2}, \\
\sum_{j=1}^{n} \gamma_{r j}=\phi_{r}, \quad \forall r \in M_{2}, \\
\sum_{j=1}^{n} \delta_{r j}^{\prime}=\theta_{r}^{\prime}, \quad \forall r \in M_{2},
\end{gathered}
$$

$\alpha_{j} \geq 0, \quad \beta_{j} \geq 0, \quad \alpha_{j}^{\prime}-\alpha_{j} \geq 0, \quad$ and $\quad \beta_{j}^{\prime}-\beta_{j} \geq 0$,

Step 7: by using Definition 7, problem (43) can be $\forall j=1,2, \ldots, n$. converted into problem (45):

$$
\operatorname{Max} / \operatorname{Min} \sum_{j=1}^{n}\left[\frac{1}{4}\left\{\left(\int_{0}^{1}\left(\omega_{j}-\sigma_{j} L^{-1}(\alpha)\right) \mathrm{d} \alpha+\int_{0}^{1}\left(\omega_{j}+\kappa_{j} R^{-1}(\alpha)\right) \mathrm{d} \alpha\right)+\left(\int_{0}^{1}\left(\omega_{j}-\sigma_{j}^{\prime} L^{\prime-1}(\alpha)\right) \mathrm{d} \alpha+\int_{0}^{1}\left(\omega_{j}+\kappa_{j}^{\prime} R^{\prime-1}(\alpha)\right) \mathrm{d} \alpha\right)\right\}\right],
$$


which subject to

$$
\begin{aligned}
& \sum_{j=1}^{n}\left(\frac{1}{4}\left\{\left(\int_{0}^{1}\left(\lambda_{l j}-\delta_{l j} L^{-1}(\alpha)\right) \mathrm{d} \alpha+\int_{0}^{1}\left(\lambda_{l j}+\gamma_{l j} R^{-1}(\alpha)\right) \mathrm{d} \alpha\right)+\left(\int_{0}^{1}\left(\lambda_{l j}-\delta_{l j}^{\prime} L^{\prime^{-1}}(\alpha)\right) \mathrm{d} \alpha+\int_{0}^{1}\left(\lambda_{l j}+\gamma_{l j}^{\prime} R^{\prime^{-1}}(\alpha)\right) \mathrm{d} \alpha\right)\right\}\right) \\
& \quad \leq \frac{1}{4}\left\{\left(\int_{0}^{1}\left(b_{l}-\theta_{l} L^{-1}(\alpha)\right) \mathrm{d} \alpha+\int_{0}^{1}\left(b_{l}+\phi_{l} R^{-1}(\alpha)\right) \mathrm{d} \alpha\right)+\left(\int_{0}^{1}\left(b_{l}-\theta_{l} L^{\prime-1}(\alpha)\right) \mathrm{d} \alpha+\int_{0}^{1}\left(b_{l}+\phi_{l} R^{\prime^{-1}}(\alpha)\right) \mathrm{d} \alpha\right)\right\}, \quad \forall l \in M_{1}, \\
& \sum_{j=1}^{n}\left(\frac{1}{4}\left\{\left(\int_{0}^{1}\left(\lambda_{s j}-\delta_{s j} L^{-1}(\alpha)\right) \mathrm{d} \alpha+\int_{0}^{1}\left(\lambda_{s j}+\gamma_{s j} R^{-1}(\alpha)\right) \mathrm{d} \alpha\right)+\left(\int_{0}^{1}\left(\lambda_{s j}-\delta_{s}^{\prime} L^{\prime-1}(\alpha)\right) \mathrm{d} \alpha+\int_{0}^{1}\left(\lambda_{s j}+\gamma_{s j}^{\prime}{R^{\prime}}^{-1}(\alpha)\right) \mathrm{d} \alpha\right)\right\}\right) \\
& \geq \frac{1}{4}\left\{\left(\int_{0}^{1}\left(b_{s}-\theta_{s} L^{-1}(\alpha)\right) \mathrm{d} \alpha+\int_{0}^{1}\left(b_{s}+\phi_{s} R^{-1}(\alpha)\right) \mathrm{d} \alpha\right)+\left(\int_{0}^{1}\left(b_{s}-\theta_{s}^{\prime} L^{\prime-1}(\alpha)\right) \mathrm{d} \alpha+\int_{0}^{1}\left(b_{s}+\phi_{s} R^{\prime-1}(\alpha)\right) \mathrm{d} \alpha\right)\right\}, \quad \forall s \in M_{3}, \\
& \sum_{j=1}^{n} \lambda_{r j}=b_{r}, \quad \forall r \in M_{2}, \\
& \sum_{j=1}^{n} \delta_{r j}=\theta_{r}, \quad \forall r \in M_{2}, \\
& \sum_{j=1}^{n} \gamma_{r j}^{\prime}=\phi_{r}^{\prime}, \quad \forall r \in M_{2}, \\
& \sum_{j=1}^{n} \gamma_{r j}=\phi_{r}, \quad \forall r \in M_{2}, \\
& \sum_{j=1}^{n} \delta_{r j}^{\prime}=\theta_{r}^{\prime}, \quad \forall r \in M_{2},
\end{aligned}
$$

$\alpha_{j} \geq 0, \quad \beta_{j} \geq 0, \quad \alpha_{j}^{\prime}-\alpha_{j} \geq 0, \quad$ and $\quad \beta_{j}^{\prime}-\beta_{j} \geq 0$, $\forall j=1,2, \ldots, n$.

Step 8: solve the crisp linear/nonlinear programming problem (45) by any existing method to find the optimal solution $x_{j}^{*}, \alpha_{j}^{*}, \beta_{j}^{*}, \alpha_{j}^{*}, \beta_{j}^{*}$.

Step 9: find the $L R$-type Pythagorean fuzzy optimal solution $X_{j}^{*}$ of the FPFLPP (13) by substituting the values of $x_{j}^{*}, \alpha_{j}^{*}, \beta_{j}^{*}, \alpha_{j}^{\prime *}$, and $\beta_{j}^{\prime *}$ in $X_{j}^{*}=\left(x_{j}^{*} ; \alpha_{j}^{*}, \beta_{j}^{*} ; \alpha_{j}^{\prime *}, \beta_{j}^{\prime *}\right)_{L R}$.

Step 10: find the $L R$-type Pythagorean fuzzy optimal value of the FPFLPP (13) by substituting the values of $X_{j}^{*}$, as calculated in Step (9), in $\sum_{j=1}^{n} C_{j} \otimes X_{j}$.

\section{Equivalence of the Proposed Methods}

Here, we confirm that the two techniques proposed in Section 3.1 and Section 3.2 give the same solution.

If $A_{1}$ and $A_{2}$ are any two PFNs such that $A_{1}=A_{2}$, then $\mathfrak{R}(A)=\mathfrak{R}(B)$. Thus, the $1^{\text {st }}$ and $2^{\text {nd }}$ constraints $\left[\sum_{j=1}^{n}\right.$
$A_{l j} \otimes X_{j} \oplus S_{l}=B_{l} \oplus S_{l}^{\prime}, \forall l \in M_{1}, \quad \sum_{j=1}^{n} A_{s j} \otimes X_{j} \oplus S_{s}=B_{s} \oplus S_{s}^{\prime}$, $\forall s \in M_{3}$ ] of Problem (19) can be written as

$$
\begin{array}{ll}
\mathfrak{R}\left(\sum_{j=1}^{n} A_{l j} \otimes X_{j} \oplus S_{l}\right)=\mathfrak{R}\left(B_{l} \oplus S_{l}^{\prime}\right), & \forall l \in M_{1}, \\
\mathfrak{R}\left(\sum_{j=1}^{n} A_{s j} \otimes X_{j} \oplus S_{s}\right)=\mathfrak{R}\left(B_{s} \oplus S_{s}^{\prime}\right), & \forall s \in M_{3} .
\end{array}
$$

Since the ranking function as discussed in Definition 7 is linear, so equations (47) and (48) can be written as

$$
\mathfrak{R}\left(\sum_{j=1}^{n} A_{l j} \otimes X_{j}\right)+\mathfrak{R}\left(S_{l}\right)=\mathfrak{R}\left(B_{l}\right)+\mathfrak{R}\left(S_{l}^{\prime}\right), \quad \forall l \in M_{1},
$$

$$
\mathfrak{R}\left(\sum_{j=1}^{n} A_{s j} \otimes X_{j}\right)+\mathfrak{R}\left(S_{s}\right)=\mathfrak{R}\left(B_{s}\right) \Re\left(S_{s}^{\prime}\right), \quad \forall s \in M_{3} .
$$


Thus, equations (49) and (50) can be written as

$$
\begin{aligned}
& \mathfrak{R}\left(B_{l}\right)-\mathfrak{R}\left(\sum_{j=1}^{n} A_{l j} \otimes X_{j}\right)=\mathfrak{R}\left(S_{l}\right)-\mathfrak{R}\left(S_{l}^{\prime}\right), \quad \forall l \in M_{1}, \\
& \mathfrak{R}\left(B_{s}\right)-\mathfrak{R}\left(\sum_{j=1}^{n} A_{s j} \otimes X_{j}\right)=\mathfrak{R}\left(S_{s}\right)-\mathfrak{R}\left(S_{s}^{\prime}\right), \quad \forall s \in M_{3} .
\end{aligned}
$$

Now, by using the $4^{\text {th }}$ and $5^{\text {th }}$ constraints $\left[\mathfrak{R}\left(S_{l}\right)-\mathfrak{R}\left(S_{l}^{\prime}\right) \geq 0, \mathfrak{R}\left(S_{s}\right)-\mathfrak{R}\left(S_{s}^{\prime}\right) \leq 0\right]$ of problem (19), equations (51) and (52) convert to

$$
\begin{aligned}
& \Re\left(B_{l}\right)-\mathfrak{R}\left(\sum_{j=1}^{n} A_{l j} \otimes X_{j}\right) \geq 0, \quad \forall l \in M_{1}, \\
& \mathfrak{R}\left(B_{s}\right)-\mathfrak{R}\left(\sum_{j=1}^{n} A_{s j} \otimes X_{j}\right) \leq 0, \quad \forall s \in M_{3},
\end{aligned}
$$

or

$$
\begin{aligned}
& \Re\left(\sum_{j=1}^{n} A_{l j} \otimes X_{j}\right) \leq \mathfrak{R}\left(B_{l}\right), \quad \forall l \in M_{1}, \\
& \mathfrak{R}\left(\sum_{j=1}^{n} A_{s j} \otimes X_{j}\right) \geq \mathfrak{R}\left(B_{s}\right), \quad \forall s \in M_{3} .
\end{aligned}
$$

Hence, the proposed techniques (method 1 and method 2) are equivalent. Both the techniques give almost the same solution. However, there is a little bit of difference that when solving the translated crisp problem, one of them may give an answer more faster than the other one. So, depending on the initial guess for the solver, technique which gives faster optimal solution is not known in advance.

\section{Numerical Examples}

Example 1. A farmer has $(45 ; 41,80 ; 44,138)_{L R}$ square-feet land. He wants to grow two types of plants, namely, $X$ and $Y$. Each $X$ plant needs $(5 ; 3,2 ; 3,4)_{L R}$ square-feet of land and $(2 ; 1,1 ; 2,3)_{L R}$ man-per-hour labor. Each $Y$ plant needs $(6 ; 5,4 ; 5,6)_{L R}$ square-feet of land and $(3 ; 2,3 ; 3,3)_{L R}$ manper-hour labor. Maximum labor which is available is $(21 ; 18,48 ; 21,74)_{L R}$ man-per-hour. Profit for each $X$ plant is $(4 ; 1,2 ; 3,4)_{L R}$ and for each $Y$ plant is $(3 ; 2,3 ; 3,5)_{L R}$. Farmer wants to maximize his profit subject to give available resources with $L(x)=R(x)=\max \left\{0,1-x^{3}\right\} \quad$ and $L^{\prime}(x)=R^{\prime}(x)=\min \left\{1, x^{2}\right\}$.

Let $X_{1}$ be the number of $X$ plants and $X_{2}$ be the number of $Y$ plants that farmer should grow. Then, the problem converts to the following LR-type FPFLPP:

$$
\operatorname{Max}(4 ; 1,2 ; 3,4)_{L R} \otimes X_{1} \oplus(3 ; 2,3 ; 3,5)_{L R} \otimes X_{2},
$$

which subject to

$$
\begin{aligned}
& (2 ; 1,1 ; 2,3)_{L R} \otimes X_{1} \oplus(3 ; 2,3 ; 3,3)_{L R} \otimes X_{2} \preccurlyeq(21 ; 18,48 ; 21,74)_{L R}, \\
& (5 ; 3,2 ; 3,4)_{L R} \otimes X_{1} \oplus(6 ; 5,4 ; 5,6)_{L R} \otimes X_{2}=(45 ; 41,80 ; 44,138)_{L R},
\end{aligned}
$$

where $X_{j}$ are $L R$-type PFNs for $j=1,2$ and $L(x)=R(x)=$ $\max \left\{0,1-x^{3}\right\}$ and $L^{\prime}(x)=R^{\prime}(x)=\min \left\{1, x^{2}\right\}$.

Now, we solve Example 1 by using method 1 as discussed in Section 3.1.

Step 1: by applying Step 2 of the presented method 1 in Section 3.1, the problem becomes
$\operatorname{Max}(4 ; 1,2 ; 3,4)_{L R} \otimes X_{1} \oplus(3 ; 2,3 ; 3,5)_{L R} \otimes X_{2}$

which subject to

$$
\begin{aligned}
(2 ; 1,1 ; 2,3)_{L R} \otimes X_{1} \oplus(3 ; 2,3 ; 3,3)_{L R} \otimes X_{2} \oplus S & =(21 ; 18,48 ; 21,74)_{L R} \oplus S^{\prime} \\
(5 ; 3,2 ; 3,4)_{L R} \otimes X_{1} \oplus(6 ; 5,4 ; 5,6)_{L R} \otimes X_{2} & =(45 ; 41,80 ; 44,138)_{L R}
\end{aligned}
$$

$\mathfrak{R}(S)-\mathfrak{R}\left(S^{\prime}\right) \geq 0$, where $X_{j}, S$, and $S^{\prime}$ are $L R$-type PFNs for $j=1,2$ and $L(x)=R(x)=\max \left\{0,1-x^{3}\right\}$ and $L^{\prime}(x)=R^{\prime}(x)=\min \left\{1, x^{2}\right\}$.

Step 2: let $X_{1}=\left(x_{1} ; \alpha_{1}, \beta_{1} ; \alpha_{1}^{\prime}, \beta_{1}^{\prime}\right)_{L R}, X_{2}=\left(x_{2} ; \alpha_{2}, \beta_{2}\right.$; $\left.\alpha_{2}^{\prime}, \beta_{2}^{\prime}\right)_{L R}, \quad S=(m ; e, f ; g, h)_{L R}, \quad$ and $S^{\prime}=\left(m^{\prime} ; e^{\prime}, f^{\prime} ;\right.$ $\left.g^{\prime}, h^{\prime}\right)_{L R}$, and the problem obtained in Step (1) can be written as

$$
\begin{aligned}
& \operatorname{Max}(4 ; 1,2 ; 3,4)_{L R} \otimes\left(x_{1} ; \alpha_{1}, \beta_{1} ; \alpha_{1}^{\prime}, \beta_{1}^{\prime}\right)_{L R} \\
& \oplus(3 ; 2,3 ; 3,5)_{L R} \otimes\left(x_{2} ; \alpha_{2}, \beta_{2} ; \alpha_{2}^{\prime}, \beta_{2}^{\prime}\right)_{L R}
\end{aligned}
$$


which subject to

$$
\begin{aligned}
(2 ; 1,1 ; 2,3)_{L R} \otimes & \left(x_{1} ; \alpha_{1}, \beta_{1} ; \alpha_{1}^{\prime}, \beta_{1}^{\prime}\right)_{L R} \oplus(3 ; 2,3 ; 3,3)_{L R} \otimes\left(x_{2} ; \alpha_{2}, \beta_{2} ; \alpha_{2}^{\prime}, \beta_{2}^{\prime}\right)_{L R} \oplus(m ; e, f ; g, h)_{L R} \\
= & (21 ; 18,48 ; 21,74)_{L R} \oplus\left(m^{\prime} ; e^{\prime}, f^{\prime} ; g^{\prime}, h^{\prime}\right)_{L R}, \\
(5 ; 3,2 ; 3,4)_{L R} \otimes & \left(x_{1} ; \alpha_{1}, \beta_{1} ; \alpha_{1}^{\prime}, \beta_{1}^{\prime}\right)_{L R} \oplus(6 ; 5,4 ; 5,6)_{L R} \otimes\left(x_{2} ; \alpha_{2}, \beta_{2} ; \alpha_{2}^{\prime}, \beta_{2}^{\prime}\right)_{L R}=(45 ; 41,80 ; 44,138)_{L R}, \\
& \Re(m ; e, f ; g, h)_{L R}-\Re\left(m^{\prime} ; e^{\prime}, f^{\prime} ; g^{\prime}, h^{\prime}\right)_{L R} \geq 0,
\end{aligned}
$$

where $\quad\left(x_{1} ; \alpha_{1}, \beta_{1} ; \alpha_{1}^{\prime}, \beta_{1}^{\prime}\right)_{L R}, \quad\left(x_{2} ; \alpha_{2}, \beta_{2} ; \alpha_{2}^{\prime}, \beta_{2}^{\prime}\right)_{L R}$, $(m ; e, f ; g, h)_{L R}$, and $\left(m^{\prime} ; e^{\prime}, f^{\prime} ; g^{\prime}, h^{\prime}\right)_{L R}$ are $L R$-type PFNs.
Step 3: using the product as discussed in Section 2, the FPFLPP, obtained in Step 2, can be written as

$$
\begin{aligned}
\operatorname{Max} & \left(4 x_{1} ; 4 x_{1}-\min \left\{3 x_{1}-3 \alpha_{1}, 6 x_{1}-6 \alpha_{1}\right\}, \max \left\{6 x_{1}+6 \beta_{1}, 3 x_{1}+3 \beta_{1}\right\}\right. \\
& \left.-4 x_{1} ; 4 x_{1}-\min \left\{x_{1}-\alpha_{1}^{\prime}, 8 x_{1}-8 \alpha_{1}^{\prime}\right\}, \max \left\{8 x_{1}+8 \beta_{1}^{\prime}, x_{1}+\beta_{1}^{\prime}\right\}-4 x_{1}\right)_{L R} \\
& \oplus\left(3 x_{2} ; 3 x_{2}-\min \left\{x_{2}-\alpha_{2}, 6 x_{2}-6 \alpha_{2}\right\}, \max \left\{6 x_{2}+6 \beta_{2}, x_{2}+\beta_{2}\right\}-3 x_{2} ;\right. \\
& \left.3 x_{2}-\min \left\{0,8 x_{2}-8 \alpha_{2}^{\prime}\right\}, \max \left\{8 x_{2}+8 \beta_{2}^{\prime}, 0\right\}-3 x_{2}\right)_{L R},
\end{aligned}
$$

which subject to

$$
\begin{aligned}
& \left(2 x_{1} ; 2 x_{1}-\min \left\{x_{1}-\alpha_{1}, 3 x_{1}-3 \alpha_{1}\right\}, \max \left\{3 x_{1}+3 \beta_{1}, x_{1}+\beta_{1}\right\}-2 x_{1} ; 2 x_{1}-\min \left\{0,5 x_{1}-5 \alpha_{1}^{\prime}\right\}, \max \left\{5 x_{1}+5 \beta_{1}^{\prime}, 0\right\}-2 x_{1}\right)_{L R} \\
& \oplus\left(3 x_{2} ; 3 x_{2}-\min \left\{x_{2}-\alpha_{2}, 6 x_{2}-6 \alpha_{2}\right\}, \max \left\{6 x_{2}+6 \beta_{2}, x_{2}+\beta_{2}\right\}-3 x_{2} ; 3 x_{2}-\min \left\{0,6 x_{2}-6 \alpha_{2}^{\prime}\right\}, \max \left\{6 x_{2}+6 \beta_{2}^{\prime}, 0\right\}-3 x_{2}\right)_{L R} \\
& \oplus(m ; e, f ; g, h)_{L R}=(21 ; 18,48 ; 21,74)_{L R} \oplus\left(m^{\prime} ; e^{\prime}, f^{\prime} ; g^{\prime}, h^{\prime}\right)_{L R}, \\
& \left(5 x_{1} ; 5 x_{1}-\min \left\{2 x_{1}-2 \alpha_{1}, 7 x_{1}-7 \alpha_{1}\right\}, \max \left\{7 x_{1}+7 \beta_{1}, 2 x_{1}+2 \beta_{1}\right\}-5 x_{1} ; 5 x_{1}-\min \left\{2 x_{1}-2 \alpha_{1}^{\prime}, 9 x_{1}-9 \alpha_{1}^{\prime}\right\},\right. \\
& \left.\max \left\{9 x_{1}+9 \beta_{1}^{\prime}, 2 x_{1}+2 \beta_{1}^{\prime}\right\}-5 x_{1}\right)_{L R} \oplus\left(6 x_{2} ; 6 x_{2}-\min \left\{x_{2}-\alpha_{2}, 10 x_{2}-10 \alpha_{2}\right\},\right. \\
& \max \left\{10 x_{2}+10 \beta_{2}, x_{2}+\beta_{2}\right\}-6 x_{2} ; 6 x_{2}-\min \left\{x_{2}-\alpha_{2}^{\prime}, 12 x_{2}-12 \alpha_{2}^{\prime}\right\}, \\
& \left.\max \left\{12 x_{2}+12 \beta_{2}^{\prime}, x_{2}+\beta_{2}^{\prime}\right\}-6 x_{2}\right)_{L R}=(45 ; 41,80 ; 44,138)_{L R}, \\
& \Re(m ; e, f ; g, h)_{L R}-\Re\left(m^{\prime} ; e^{\prime}, f^{\prime} ; g^{\prime}, h^{\prime}\right)_{L R} \geq 0,
\end{aligned}
$$

where $\left(x_{1} ; \alpha_{1}, \beta_{1} ; \alpha_{1}^{\prime}, \beta_{1}^{\prime}\right)_{L R}, \quad\left(x_{2} ; \alpha_{2}, \beta_{2} ; \alpha_{2}^{\prime}, \beta_{2}^{\prime}\right)_{L R}, \quad(m ; e$, $f ; g, h)_{L R}$, and $\left(m^{\prime} ; e^{\prime}, f^{\prime} ; g^{\prime}, h^{\prime}\right)_{L R}$ are LR-type PFNs.
Step 4: by using arithmetic operations discussed in Section 2 and using Definition 6, the FPFLPP, obtained in Step 3, can be rewritten as

$$
\begin{aligned}
& \operatorname{Max}\left(4 x_{1}+3 x_{2} ; 4 x_{1}-\min \left\{3 x_{1}-3 \alpha_{1}, 6 x_{1}-6 \alpha_{1}\right\}+3 x_{2}-\min \left\{x_{2}-\alpha_{2}, 6 x_{2}-6 \alpha_{2}\right\},\right. \\
& \max \left\{6 x_{1}+6 \beta_{1}, 3 x_{1}+3 \beta_{1}\right\}-4 x_{1}+\max \left\{6 x_{2}+6 \beta_{2}, x_{2}+\beta_{2}\right\}-3 x_{2} ; 4 x_{1} \\
& -\min \left\{x_{1}-\alpha_{1}^{\prime}, 8 x_{1}-8 \alpha_{1}^{\prime}\right\}+3 x_{2}-\min \left\{0,8 x_{2}-8 \alpha_{2}^{\prime}\right\}, \\
& \left.\max \left\{8 x_{1}+8 \beta_{1}^{\prime}, x_{1}+\beta_{1}^{\prime}\right\}-4 x_{1}+\max \left\{8 x_{2}+8 \beta_{2}^{\prime}, 0\right\}-3 x_{2}\right)_{L R},
\end{aligned}
$$


which subject to

$$
\begin{aligned}
2 x_{1}+3 x_{2}+m & =21+m^{\prime}, \\
5 x_{1}+6 x_{2} & =45, \\
2 x_{1}-\min \left\{x_{1}-\alpha_{1}, 3 x_{1}-3 \alpha_{1}\right\}+3 x_{2}-\min \left\{x_{2}-\alpha_{2}, 6 x_{2}-6 \alpha_{2}\right\}+e & =18+e^{\prime}, \\
\max \left\{3 x_{1}+3 \beta_{1}, x_{1}+\beta_{1}\right\}-2 x_{1}+\max \left\{6 x_{2}+6 \beta_{2}, x_{2}+\beta_{2}\right\}-3 x_{2}+f & =48+f^{\prime}, \\
2 x_{1}-\min \left\{0,5 x_{1}-5 \alpha_{1}^{\prime}\right\}+3 x_{2}-\min \left\{0,6 x_{2}-6 \alpha_{2}^{\prime}\right\}+g & =21+g^{\prime}, \\
\max \left\{5 x_{1}+5 \beta_{1}^{\prime}, 0\right\}-2 x_{1}+\max \left\{6 x_{2}+6 \beta_{2}^{\prime}, 0\right\}-3 x_{2}+h & =74+h^{\prime}, \\
5 x_{1}-\min \left\{2 x_{1}-2 \alpha_{1}, 7 x_{1}-7 \alpha_{1}\right\}+6 x_{2}-\min \left\{x_{2}-\alpha_{2}, 10 x_{2}-10 \alpha_{2}\right\} & =41, \\
\max \left\{7 x_{1}+7 \beta_{1}, 2 x_{1}+2 \beta_{1}\right\}-5 x_{1}+\max \left\{10 x_{2}+10 \beta_{2}, x_{2}+\beta_{2}\right\}-6 x_{2} & =80, \\
5 x_{1}-\min \left\{2 x_{1}-2 \alpha_{1}^{\prime}, 9 x_{1}-9 \alpha_{1}^{\prime}\right\}+6 x_{2}-\min \left\{x_{2}-\alpha_{2}^{\prime}, 12 x_{2}-12 \alpha_{2}^{\prime}\right\} & =44, \\
\max \left\{9 x_{1}+9 \beta_{1}^{\prime}, 2 x_{1}+2 \beta_{1}^{\prime}\right\}-5 x_{1}+\max \left\{12 x_{2}+12 \beta_{2}^{\prime}, x_{2}+\beta_{2}^{\prime}\right\}-6 x_{2} & =138,
\end{aligned}
$$

$\mathfrak{R}(m ; e, f ; g, h)_{L R}-\mathfrak{R}\left(m^{\prime} ; e^{\prime}, f^{\prime} ; g^{\prime}, h^{\prime}\right)_{L R} \geq 0$, where $\left(x_{1} ; \alpha_{1}, \beta_{1} ; \alpha_{1}^{\prime}, \beta_{1}^{\prime}\right)_{L R}, \quad\left(x_{2} ; \alpha_{2}, \beta_{2} ; \alpha_{2}^{\prime}, \beta_{2}^{\prime}\right)_{L R}, \quad(m ; e, f ; g$, $h)_{L R}$, and $\left(m^{\prime} ; e^{\prime}, f^{\prime} ; g^{\prime}, h^{\prime}\right)_{L R}$ are LR-type PFNs.
Step 5: using Step 6 of the proposed method 1, the FPFLPP, obtained in Step 4, can be rewritten as

$$
\begin{aligned}
& \operatorname{Max} \Re\left(4 x_{1}+3 x_{2} ; 4 x_{1}-\min \left\{3 x_{1}-3 \alpha_{1}, 6 x_{1}-6 \alpha_{1}\right\}+3 x_{2}-\min \left\{x_{2}-\alpha_{2}, 6 x_{2}-6 \alpha_{2}\right\},\right. \\
& \max \left\{6 x_{1}+6 \beta_{1}, 3 x_{1}+3 \beta_{1}\right\}-4 x_{1}+\max \left\{6 x_{2}+6 \beta_{2}, x_{2}+\beta_{2}\right\}-3 x_{2} ; 4 x_{1} \\
& -\min \left\{x_{1}-\alpha_{1}^{\prime}, 8 x_{1}-8 \alpha_{1}^{\prime}\right\}+3 x_{2}-\min \left\{0,8 x_{2}-8 \alpha_{2}^{\prime}\right\}, \\
& \left.\max \left\{8 x_{1}+8 \beta_{1}^{\prime}, x_{1}+\beta_{1}^{\prime}\right\}-4 x_{1}+\max \left\{8 x_{2}+8 \beta_{2}^{\prime}, 0\right\}-3 x_{2}\right)_{L R},
\end{aligned}
$$

which subject to

$$
\begin{aligned}
2 x_{1}+3 x_{2}+m & =21+m^{\prime}, \\
5 x_{1}+6 x_{2} & =45, \\
2 x_{1}-\min \left\{x_{1}-\alpha_{1}, 3 x_{1}-3 \alpha_{1}\right\}+3 x_{2}-\min \left\{x_{2}-\alpha_{2}, 6 x_{2}-6 \alpha_{2}\right\}+e & =18+e^{\prime}, \\
\max \left\{3 x_{1}+3 \beta_{1}, x_{1}+\beta_{1}\right\}-2 x_{1}+\max \left\{6 x_{2}+6 \beta_{2}, x_{2}+\beta_{2}\right\}-3 x_{2}+f & =48+f^{\prime}, \\
2 x_{1}-\min \left\{0,5 x_{1}-5 \alpha_{1}^{\prime}\right\}+3 x_{2}-\min \left\{0,6 x_{2}-6 \alpha_{2}^{\prime}\right\}+g & =21+g^{\prime}, \\
\max \left\{5 x_{1}+5 \beta_{1}^{\prime}, 0\right\}-2 x_{1}+\max \left\{6 x_{2}+6 \beta_{2}^{\prime}, 0\right\}-3 x_{2}+h & =74+h^{\prime}, \\
5 x_{1}-\min \left\{2 x_{1}-2 \alpha_{1}, 7 x_{1}-7 \alpha_{1}\right\}+6 x_{2}-\min \left\{x_{2}-\alpha_{2}, 10 x_{2}-10 \alpha_{2}\right\} & =41, \\
\max \left\{7 x_{1}+7 \beta_{1}, 2 x_{1}+2 \beta_{1}\right\}-5 x_{1}+\max \left\{10 x_{2}+10 \beta_{2}, x_{2}+\beta_{2}\right\}-6 x_{2} & =80, \\
5 x_{1}-\min \left\{2 x_{1}-2 \alpha_{1}^{\prime}, 9 x_{1}-9 \alpha_{1}^{\prime}\right\}+6 x_{2}-\min \left\{x_{2}-\alpha_{2}^{\prime}, 12 x_{2}-12 \alpha_{2}^{\prime}\right\} & =44, \\
\max \left\{9 x_{1}+9 \beta_{1}^{\prime}, 2 x_{1}+2 \beta_{1}^{\prime}\right\}-5 x_{1}+\max \left\{12 x_{2}+12 \beta_{2}^{\prime}, x_{2}+\beta_{2}^{\prime}\right\}-6 x_{2} & =138,
\end{aligned}
$$

$\Re(m ; e, f ; g, h)_{L R}-\Re\left(m^{\prime} ; e^{\prime}, f^{\prime} ; g^{\prime}, h^{\prime}\right)_{L R} \geq 0, \alpha_{1} \geq 0$, $\beta_{1} \geq 0, \alpha_{1}^{\prime}-\alpha_{1} \geq 0, \beta_{1}^{\prime}-\beta_{1} \geq 0, \alpha_{2} \geq 0, \beta_{2} \geq 0, \alpha_{2}^{\prime}-\alpha_{2} \geq 0$, $\beta_{2}^{\prime}-\beta_{2} \geq 0, e \geq 0, f \geq 0, g-e \geq 0, h-f \geq 0, e^{\prime} \geq 0, f^{\prime} \geq$ $0, g^{\prime}-e^{\prime} \geq 0$, and $h^{\prime}-f^{\prime} \geq 0$.
Step 6: using $\min \{a, b\}=((a+b) / 2)-|(a-b) / 2|$ and $\max \{a, b\}=((a+b) / 2)+|(a-b) / 2|$ and Steps 8 and 9 of method 1, presented in Section 3.1, the FPFLPP, obtained in Step 5, can be written as 


$$
\begin{aligned}
\operatorname{Max} & \left(\frac{65}{12} x_{1}+\frac{61}{12} x_{2}-\frac{27}{8} \alpha_{1}-\frac{9}{8}\left|x_{1}-\alpha_{1}\right|-\frac{21}{8} \alpha_{2}-\frac{15}{8}\left|x_{2}-\alpha_{2}\right|+\frac{27}{8} \beta_{1}+\frac{9}{8}\left|x_{1}+\beta_{1}\right|+\frac{21}{8} \beta_{2}\right. \\
& \left.+\frac{15}{8}\left|x_{2}+\beta_{2}\right|-3 \alpha_{1}^{\prime}-\frac{7}{3}\left|x_{1}-\alpha_{1}^{\prime}\right|-\frac{8}{3} \alpha_{2}^{\prime}-\frac{8}{3}\left|x_{2}-\alpha_{2}^{\prime}\right|+3 \beta_{1}^{\prime}+\frac{7}{3}\left|x_{1}+\beta_{1}^{\prime}\right|+\frac{8}{3} \beta_{2}^{\prime}+\frac{8}{3}\left|x_{2}+\beta_{2}^{\prime}\right|\right)
\end{aligned}
$$

which subject to

$$
\begin{aligned}
2 x_{1}+3 x_{2}+m & =21+m^{\prime}, \\
5 x_{1}+6 x_{2} & =45, \\
2 \alpha_{1}+\left|x_{1}-\alpha_{1}\right|-\frac{1}{2} x_{2}+\frac{7}{2} \alpha_{2}+\frac{5}{2}\left|x_{2}-\alpha_{2}\right|+e & =18+e^{\prime} \\
2 \beta_{1}+\left|x_{1}+\beta_{1}\right|+\frac{1}{2} x_{2}+\frac{7}{2} \beta_{2}+\frac{5}{2}\left|x_{2}+\beta_{2}\right|+f & =48+f^{\prime}, \\
-\frac{1}{2} x_{1}+\frac{5}{2} \alpha_{1}^{\prime}+\frac{5}{2}\left|x_{1}-\alpha_{1}^{\prime}\right|+3 \alpha_{2}^{\prime}+3\left|x_{2}-\alpha_{2}^{\prime}\right|+g & =21+g^{\prime}, \\
\frac{1}{2} x_{1}+\frac{5}{2} \beta_{1}^{\prime}+\frac{5}{2}\left|x_{1}+\beta_{1}^{\prime}\right|+3 \beta_{2}^{\prime}+3\left|x_{2}+\beta_{2}^{\prime}\right|+h & =74+h^{\prime}, \\
\frac{1}{2} x_{1}+\frac{9}{2} \alpha_{1}+\frac{5}{2}\left|x_{1}-\alpha_{1}\right|+\frac{1}{2} x_{2}+\frac{11}{2} \alpha_{2}+\frac{9}{2}\left|x_{2}-\alpha_{2}\right| & =41 \\
-\frac{1}{2} x_{1}+\frac{9}{2} \beta_{1}+\frac{5}{2}\left|x_{1}+\beta_{1}\right|-\frac{1}{2} x_{2}+\frac{11}{2} \beta_{2}+\frac{9}{2}\left|x_{2}+\beta_{2}\right| & =80 \\
\frac{1}{1} x_{1}+\frac{11}{2} \alpha_{1}^{\prime}+\frac{7}{2}\left|x_{1}-\alpha_{1}^{\prime}\right|-\frac{1}{2} x_{2}+\frac{13}{2} \alpha_{2}^{\prime}+\frac{11}{2}\left|x_{2}-\alpha_{2}^{\prime}\right| & =44 \\
\frac{1}{2} x_{1}+\frac{11}{2} \beta_{1}^{\prime}+\frac{7}{2}\left|x_{1}+\beta_{1}^{\prime}\right|+\frac{1}{2} x_{2}+\frac{13}{2} \beta_{2}^{\prime}+\frac{11}{2}\left|x_{2}+\beta_{2}^{\prime}\right| & =138 \\
\frac{8}{2}+f-g+h-8 m^{\prime}+e^{\prime}-f^{\prime}+g^{\prime}-h^{\prime} & \geq 0
\end{aligned}
$$

$\alpha_{1} \geq 0, \beta_{1} \geq 0, \alpha_{1}^{\prime}-\alpha_{1} \geq 0, \beta_{1}^{\prime}-\beta_{1} \geq 0, \alpha_{2} \geq 0, \beta_{2} \geq 0, \alpha_{2}^{\prime}-$ $\alpha_{2} \geq 0, \beta_{2}^{\prime}-\beta_{2} \geq 0, e \geq 0, f \geq 0, g-e \geq 0, h-f \geq 0, e^{\prime} \geq$ $0, f^{\prime} \geq 0, g^{\prime}-e^{\prime} \geq 0$, and $h^{\prime}-f^{\prime} \geq 0$.

Step 7: the optimal solution of the crisp nonlinear programming problem, obtained in Step 5(using: MATLAB R2014a, solver "fmincon," algorithm "interior point," TolFun =eps, TolX = eps, TolCon $=0.000001)$ is $x_{1}=9.0001, \alpha_{1}=0.001, \beta_{1}=8.857$, $\alpha_{1}^{\prime}=0.1011, \beta_{1}^{\prime}=11.3333, x_{2}=-0.0001, \alpha_{2}=1.3996$, $\beta_{2}=0.0001, \alpha_{2}^{\prime}=1.3997$, and $\beta_{2}^{\prime}=0.0002$.

Step 8: substituting the values of $x_{1}, \alpha_{1}, \beta_{1}, \alpha_{1}^{\prime}$, $\beta_{1}^{\prime}, x_{2}, \alpha_{2}, \beta_{2}, \alpha_{2}^{\prime}$, and $\beta_{2}^{\prime}$ in $X_{1}=\left(x_{1} ; \alpha_{1}, \beta_{1} ; \alpha_{1}^{\prime}, \beta_{1}^{\prime}\right)_{L R}$ and $X_{2}=\left(x_{2} ; \alpha_{2}, \beta_{2} ; \alpha_{2}^{\prime}, \beta_{2}^{\prime}\right)_{L R}$, the exact LR-type Pythagorean fuzzy optimal solution is $X_{1}=$ $(9.0001 ; 0.001,8.8570 .1011,11.3333)_{L R}, \quad$ and $\quad X_{2}=$ $(-0.0001 ; 1.3996,0.0001 ; 1.3997,0.0002)_{L R}$.
Step 9: substituting the values of $X_{1}$ and $X_{2}$, obtained in Step 8, into the objective function, the LR-type Pythagorean fuzzy optimal value is (36.0001; $9.0034,75.3404 ; 38.2995,126.6415)_{L R}$. So, the farmer should grow $(9.0001 ; 0.001,8.857 ; 0.1011,11.3333)_{L R}$ number of plants of $X$ and $(-0.0001 ; 1.3996,0.0001$; $1.3997,0.0002)_{L R}$ number of plants of $Y$ to get a maximum profit of $(36.0001 ; 9.0034,75.3404$; $38.2995,126.6415)_{L R}$.

We now solve Example 1 by using method 2 as discussed in Section 3.2:

Step 1: let $X_{1}=\left(x_{1} ; \alpha_{1}, \beta_{1} ; \alpha_{1}^{\prime}, \beta_{1}^{\prime}\right)_{L R}$ and $X_{2}=\left(x_{2} ; \alpha_{2}\right.$, $\left.\beta_{2} ; \alpha_{2}^{\prime}, \beta_{2}^{\prime}\right)_{L R}$; then, problem of Example 1 can be written as 
which subject to

$$
\begin{aligned}
& (2 ; 1,1 ; 2,3)_{L R} \otimes\left(x_{1} ; \alpha_{1}, \beta_{1} ; \alpha_{1}^{\prime}, \beta_{1}^{\prime}\right)_{L R} \oplus(3 ; 2,3 ; 3,3)_{L R} \otimes\left(x_{2} ; \alpha_{2}, \beta_{2} ; \alpha_{2}^{\prime}, \beta_{2}^{\prime}\right)_{L R} \preccurlyeq(21 ; 18,48 ; 21,74)_{L R}, \\
& (5 ; 3,2 ; 3,4)_{L R} \otimes\left(x_{1} ; \alpha_{1}, \beta_{1} ; \alpha_{1}^{\prime}, \beta_{1}^{\prime}\right)_{L R} \oplus(6 ; 5,4 ; 5,6)_{L R} \otimes\left(x_{2} ; \alpha_{2}, \beta_{2} ; \alpha_{2}^{\prime}, \beta_{2}^{\prime}\right)_{L R}=(45 ; 41,80 ; 44,138)_{L R},
\end{aligned}
$$

where $\left(x_{1} ; \alpha_{1}, \beta_{1} ; \alpha_{1}^{\prime}, \beta_{1}^{\prime}\right)_{L R}$ and $\left(x_{2} ; \alpha_{2}, \beta_{2} ; \alpha_{2}^{\prime}, \beta_{2}^{\prime}\right)_{L R}$ are $L R$-type PFNs.
Step 2: using the product as discussed in Section 2, the FPFLPP, obtained in Step 1, can be written as

$$
\begin{aligned}
& \operatorname{Max}\left(4 x_{1} ; 4 x_{1}-\min \left\{3 x_{1}-3 \alpha_{1}, 6 x_{1}-6 \alpha_{1}\right\}, \max \left\{6 x_{1}+6 \beta_{1}, 3 x_{1}+3 \beta_{1}\right\}-4 x_{1} ; 4 x_{1}-\min \left\{x_{1}-\alpha_{1}^{\prime}, 8 x_{1}-8 \alpha_{1}^{\prime}\right\},\right. \\
& \left.\max \left\{8 x_{1}+8 \beta_{1}^{\prime}, x_{1}+\beta_{1}^{\prime}\right\}-4 x_{1}\right)_{L R} \oplus\left(3 x_{2} ; 3 x_{2}-\min \left\{x_{2}-\alpha_{2}, 6 x_{2}-6 \alpha_{2}\right\},\right. \\
& \left.\max \left\{6 x_{2}+6 \beta_{2}, x_{2}+\beta_{2}\right\}-3 x_{2} ; 3 x_{2}-\min \left\{0,8 x_{2}-8 \alpha_{2}^{\prime}\right\}, \max \left\{8 x_{2}+8 \beta_{2}^{\prime}, 0\right\}-3 x_{2}\right)_{L R},
\end{aligned}
$$

which subject to

$$
\begin{aligned}
& \left(2 x_{1} ; 2 x_{1}-\min \left\{x_{1}-\alpha_{1}, 3 x_{1}-3 \alpha_{1}\right\}, \max \left\{3 x_{1}+3 \beta_{1}, x_{1}+\beta_{1}\right\}-2 x_{1} ; 2 x_{1}-\min \left\{0,5 x_{1}-5 \alpha_{1}^{\prime}\right\},\right. \\
& \left.\quad \max \left\{5 x_{1}+5 \beta_{1}^{\prime}, 0\right\}-2 x_{1}\right)_{L R} \oplus\left(3 x_{2} ; 3 x_{2}-\min \left\{x_{2}-\alpha_{2}, 6 x_{2}-6 \alpha_{2}\right\},\right. \\
& \left.\max \left\{6 x_{2}+6 \beta_{2}, x_{2}+\beta_{2}\right\}-3 x_{2} ; 3 x_{2}-\min \left\{0,6 x_{2}-6 \alpha_{2}^{\prime}\right\}, \max \left\{6 x_{2}+6 \beta_{2}^{\prime}, 0\right\}-3 x_{2}\right)_{L R} \leqslant(21 ; 18,48 ; 21,74)_{L R}, \\
& \left(5 x_{1} ; 5 x_{1}-\min \left\{2 x_{1}-2 \alpha_{1}, 7 x_{1}-7 \alpha_{1}\right\}, \max \left\{7 x_{1}+7 \beta_{1}, 2 x_{1}+2 \beta_{1}\right\}-5 x_{1} ; 5 x_{1}\right. \\
& \left.-\min \left\{2 x_{1}-2 \alpha_{1}^{\prime}, 9 x_{1}-9 \alpha_{1}^{\prime}\right\}, \max \left\{9 x_{1}+9 \beta_{1}^{\prime}, 2 x_{1}+2 \beta_{1}^{\prime}\right\}-5 x_{1}\right)_{L R} \\
& \oplus\left(6 x_{2} ; 6 x_{2}-\min \left\{x_{2}-\alpha_{2}, 10 x_{2}-10 \alpha_{2}\right\}, \max \left\{10 x_{2}+10 \beta_{2}, x_{2}+\beta_{2}\right\}-6 x_{2} ;\right. \\
& \left.6 x_{2}-\min \left\{x_{2}-\alpha_{2}^{\prime}, 12 x_{2}-12 \alpha_{2}^{\prime}\right\}, \max \left\{12 x_{2}+12 \beta_{2}^{\prime}, x_{2}+\beta_{2}^{\prime}\right\}-6 x_{2}\right)_{L R}=(45 ; 41,80 ; 44,138)_{L R},
\end{aligned}
$$

where $\left(x_{1} ; \alpha_{1}, \beta_{1} ; \alpha_{1}^{\prime}, \beta_{1}^{\prime}\right)_{L R}$ and $\left(x_{2} ; \alpha_{2}, \beta_{2} ; \alpha_{2}^{\prime}, \beta_{2}^{\prime}\right)_{L R}$ are $L R$-type PFNs.
Step 3: by using arithmetic operations discussed in Section 2 and Definition 6, the FPFLPP, obtained in Step 2, can be rewritten as

$$
\begin{aligned}
& \operatorname{Max}\left(4 x_{1}+3 x_{2} ; 4 x_{1}-\min \left\{3 x_{1}-3 \alpha_{1}, 6 x_{1}-6 \alpha_{1}\right\}+3 x_{2}-\min \left\{x_{2}-\alpha_{2}, 6 x_{2}-6 \alpha_{2}\right\},\right. \\
& \quad \max \left\{6 x_{1}+6 \beta_{1}, 3 x_{1}+3 \beta_{1}\right\}-4 x_{1}+\max \left\{6 x_{2}+6 \beta_{2}, x_{2}+\beta_{2}\right\}-3 x_{2} ; \\
& 4 x_{1}-\min \left\{x_{1}-\alpha_{1}^{\prime}, 8 x_{1}-8 \alpha_{1}^{\prime}\right\}+3 x_{2}-\min \left\{0,8 x_{2}-8 \alpha_{2}^{\prime}\right\}, \max \left\{8 x_{1}+8 \beta_{1}^{\prime}, x_{1}+\beta_{1}^{\prime}\right\}-4 x_{1} \\
& \left.+\max \left\{8 x_{2}+8 \beta_{2}^{\prime}, 0\right\}-3 x_{2}\right)_{L R},
\end{aligned}
$$

which subject to

$$
\begin{aligned}
& \left(2 x_{1}+3 x_{2} ; 2 x_{1}-\min \left\{x_{1}-\alpha_{1}, 3 x_{1}-3 \alpha_{1}\right\}+3 x_{2}-\min \left\{x_{2}-\alpha_{2}, 6 x_{2}-6 \alpha_{2}\right\}, \max \left\{3 x_{1}+3 \beta_{1}, x_{1}+\beta_{1}\right\}-2 x_{1}\right. \\
& +\max \left\{6 x_{2}+6 \beta_{2}, x_{2}+\beta_{2}\right\}-3 x_{2} ; 2 x_{1}-\min \left\{0,5 x_{1}-5 \alpha_{1}^{\prime}\right\}+3 x_{2}-\min \left\{0,6 x_{2}-6 \alpha_{2}^{\prime}\right\}, \\
& \left.\max \left\{5 x_{1}+5 \beta_{1}^{\prime}, 0\right\}-2 x_{1}+\max \left\{6 x_{2}+6 \beta_{2}^{\prime}, 0\right\}-3 x_{2}\right)_{L R} \leqslant(21 ; 18,48 ; 21,74)_{L R} \text {, } \\
& 5 x_{1}-\min \left\{2 x_{1}-2 \alpha_{1}, 7 x_{1}-7 \alpha_{1}\right\}+6 x_{2}-\min \left\{x_{2}-\alpha_{2}, 10 x_{2}-10 \alpha_{2}\right\}=41 \text {, } \\
& \max \left\{7 x_{1}+7 \beta_{1}, 2 x_{1}+2 \beta_{1}\right\}-5 x_{1}+\max \left\{10 x_{2}+10 \beta_{2}, x_{2}+\beta_{2}\right\}-6 x_{2}=80 \text {, } \\
& 5 x_{1}-\min \left\{2 x_{1}-2 \alpha_{1}^{\prime}, 9 x_{1}-9 \alpha_{1}^{\prime}\right\}+6 x_{2}-\min \left\{x_{2}-\alpha_{2}^{\prime}, 12 x_{2}-12 \alpha_{2}^{\prime}\right\}=44 \text {, } \\
& \max \left\{9 x_{1}+9 \beta_{1}^{\prime}, 2 x_{1}+2 \beta_{1}^{\prime}\right\}-5 x_{1}+\max \left\{12 x_{2}+12 \beta_{2}^{\prime}, x_{2}+\beta_{2}^{\prime}\right\}-6 x_{2}=138 \text {, }
\end{aligned}
$$


$5 x_{1}+6 x_{2}=45$, where $\left(x_{1} ; \alpha_{1}, \beta_{1} ; \alpha_{1}^{\prime}, \beta_{1}^{\prime}\right)_{L R} \quad$ and $\left(x_{2} ; \alpha_{2}, \beta_{2} ; \alpha_{2}^{\prime}, \beta_{2}^{\prime}\right)_{L R}$ are LR-type PFNs.
Step 4: using Step 4 of the proposed method 2, the FPFLPP, obtained in Step 3, can be rewritten as

$$
\begin{aligned}
& \operatorname{Max} \Re\left(4 x_{1}+3 x_{2} ; 4 x_{1}-\min \left\{3 x_{1}-3 \alpha_{1}, 6 x_{1}-6 \alpha_{1}\right\}+3 x_{2}-\min \left\{x_{2}-\alpha_{2}, 6 x_{2}-6 \alpha_{2}\right\},\right. \\
& \max \left\{6 x_{1}+6 \beta_{1}, 3 x_{1}+3 \beta_{1}\right\}-4 x_{1}+\max \left\{6 x_{2}+6 \beta_{2}, x_{2}+\beta_{2}\right\}-3 x_{2}, \\
& 4 x_{1}-\min \left\{x_{1}-\alpha_{1}^{\prime}, 8 x_{1}-8 \alpha_{1}^{\prime}\right\}+3 x_{2}-\min \left\{0,8 x_{2}-8 \alpha_{2}^{\prime}\right\}, \\
& \left.\max \left\{8 x_{1}+8 \beta_{1}^{\prime}, x_{1}+\beta_{1}^{\prime}\right\}-4 x_{1}+\max \left\{8 x_{2}+8 \beta_{2}^{\prime}, 0\right\}-3 x_{2}\right)_{L R},
\end{aligned}
$$

which subject to

$$
\begin{aligned}
& \Re\left(2 x_{1}+3 x_{2} ; 2 x_{1}-\min \left\{x_{1}-\alpha_{1}, 3 x_{1}-3 \alpha_{1}\right\}+3 x_{2}-\min \left\{x_{2}-\alpha_{2}, 6 x_{2}-6 \alpha_{2}\right\},\right. \\
& \max \left\{3 x_{1}+3 \beta_{1}, x_{1}+\beta_{1}\right\}-2 x_{1}+\max \left\{6 x_{2}+6 \beta_{2}, x_{2}+\beta_{2}\right\}-3 x_{2} ; 2 x_{1} \\
& \quad-\min \left\{0,5 x_{1}-5 \alpha_{1}^{\prime}\right\}+3 x_{2}-\min \left\{0,6 x_{2}-6 \alpha_{2}^{\prime}\right\}, \max \left\{5 x_{1}+5 \beta_{1}^{\prime}, 0\right\} \\
& \left.-2 x_{1}+\max \left\{6 x_{2}+6 \beta_{2}^{\prime}, 0\right\}-3 x_{2}\right)_{L R} \leq \Re(21 ; 18,48 ; 21,74)_{L R}, \\
& 5 x_{1}-\min \left\{2 x_{1}-2 \alpha_{1}, 7 x_{1}-7 \alpha_{1}\right\}+6 x_{2}-\min \left\{x_{2}-\alpha_{2}, 10 x_{2}-10 \alpha_{2}\right\}=41, \\
& \max \left\{7 x_{1}+7 \beta_{1}, 2 x_{1}+2 \beta_{1}\right\}-5 x_{1}+\max \left\{10 x_{2}+10 \beta_{2}, x_{2}+\beta_{2}\right\}-6 x_{2}=80, \\
& 5 x_{1}-\min \left\{2 x_{1}-2 \alpha_{1}^{\prime}, 9 x_{1}-9 \alpha_{1}^{\prime}\right\}+6 x_{2}-\min \left\{x_{2}-\alpha_{2}^{\prime}, 12 x_{2}-12 \alpha_{2}^{\prime}\right\}=44, \\
& \max \left\{9 x_{1}+9 \beta_{1}^{\prime}, 2 x_{1}+2 \beta_{1}^{\prime}\right\}-5 x_{1}+\max \left\{12 x_{2}+12 \beta_{2}^{\prime}, x_{2}+\beta_{2}^{\prime}\right\}-6 x_{2}=138,
\end{aligned}
$$

$5 x_{1}+6 x_{2}=45, \quad \alpha_{1} \geq 0, \beta_{1} \geq 0, \alpha_{1}^{\prime}-\alpha_{1} \geq 0, \beta_{1}^{\prime}-\beta_{1} \geq 0$, $\alpha_{2} \geq 0, \beta_{2} \geq 0, \alpha_{2}^{\prime}-\alpha_{2} \geq 0$, and $\beta_{2}^{\prime}-\beta_{2} \geq 0$.

Step 5: using $\min \{a, b\}=((a+b) / 2)-|(a-b) / 2|$ and $\max \{a, b\}=((a+b) / 2)+|(a-b) / 2|$ and Steps 6 and 7 of the presented method 2, the FPFLPP, obtained in Step 4, can be written as

$$
\begin{aligned}
& \operatorname{Max}\left(\frac{65}{12} x_{1}+\frac{61}{12} x_{2}-\frac{27}{8} \alpha_{1}-\frac{9}{8}\left|x_{1}-\alpha_{1}\right|-\frac{21}{8} \alpha_{2}-\frac{15}{8}\left|x_{2}-\alpha_{2}\right|+\frac{27}{8} \beta_{1}+\frac{9}{8}\left|x_{1}+\beta_{1}\right|+\frac{21}{8} \beta_{2}+\frac{15}{8}\left|x_{2}+\beta_{2}\right|\right. \\
& \left.-3 \alpha_{1}^{\prime}-\frac{7}{3}\left|x_{1}-\alpha_{1}^{\prime}\right|-\frac{8}{3} \alpha_{2}^{\prime}-\frac{8}{3}\left|x_{2}-\alpha_{2}^{\prime}\right|+3 \beta_{1}^{\prime}+\frac{7}{3}\left|x_{1}+\beta_{1}^{\prime}\right|+\frac{8}{3} \beta_{2}^{\prime}+\frac{8}{3}\left|x_{2}+\beta_{2}^{\prime}\right|\right),
\end{aligned}
$$


which subject to

$$
\begin{aligned}
& \frac{8}{3} x_{1}+\frac{15}{4} x_{2}-\frac{3}{2} \alpha_{1}-\frac{3}{4}\left|x_{1}-\alpha_{1}\right|-\frac{21}{8} \alpha_{2}-\frac{15}{8}\left|x_{2}-\alpha_{2}\right|+\frac{3}{2} \beta_{1}+\frac{3}{4}\left|x_{1}+\beta_{1}\right|+\frac{21}{8} \beta_{2}+\frac{15}{8} \\
& \left|x_{2}+\beta_{2}\right|-\frac{5}{3} \alpha_{1}^{\prime}-\frac{5}{3}\left|x_{1}-\alpha_{1}^{\prime}\right|-2 \alpha_{2}^{\prime}-2\left|x_{2}-\alpha_{2}^{\prime}\right|+\frac{5}{3} \beta_{1}^{\prime}+\frac{5}{3}\left|x_{1}+\beta_{1}^{\prime}\right|+2 \beta_{2}^{\prime}+2\left|x_{2}+\beta_{2}^{\prime}\right| \leq 35.4583 \\
& \frac{1}{2} x_{1}+\frac{9}{2} \alpha_{1}+\frac{5}{2}\left|x_{1}-\alpha_{1}\right|+\frac{1}{2} x_{2}+\frac{11}{2} \alpha_{2}+\frac{9}{2}\left|x_{2}-\alpha_{2}\right|=41 \\
& -\frac{1}{2} x_{1}+\frac{9}{2} \beta_{1}+\frac{5}{2}\left|x_{1}+\beta_{1}\right|-\frac{1}{2} x_{2}+\frac{11}{2} \beta_{2}+\frac{9}{2}\left|x_{2}+\beta_{2}\right|=80 \\
& -\frac{1}{2} x_{1}+\frac{11}{2} \alpha_{1}^{\prime}+\frac{7}{2}\left|x_{1}-\alpha_{1}^{\prime}\right|-\frac{1}{2} x_{2}+\frac{13}{2} \alpha_{2}^{\prime}+\frac{11}{2}\left|x_{2}-\alpha_{2}^{\prime}\right|=44 \\
& \frac{1}{2} x_{1}+\frac{11}{2} \beta_{1}^{\prime}+\frac{7}{2}\left|x_{1}+\beta_{1}^{\prime}\right|+\frac{1}{2} x_{2}+\frac{13}{2} \beta_{2}^{\prime}+\frac{11}{2}\left|x_{2}+\beta_{2}^{\prime}\right|=138 \\
& 5 x_{1}+6 x_{2}=45
\end{aligned}
$$

$\alpha_{1} \geq 0, \beta_{1} \geq 0, \alpha_{1}^{\prime}-\alpha_{1} \geq 0, \beta_{1}^{\prime}-\beta_{1} \geq 0, \alpha_{2} \geq 0, \beta_{2} \geq 0, \alpha_{2}^{\prime}-$ $\alpha_{2} \geq 0$, and $\beta_{2}^{\prime}-\beta_{2} \geq 0$.

Step 6: the optimal solution of the crisp nonlinear programming problem, obtained in Step 5(using MATLAB R2014a, solver "fmincon," algorithm "interior point," TolFun $=1$, TolX $=$ eps, TolCon $=1$ ), is $x_{1}=9, \alpha_{1}=0, \beta_{1}=8.8, \alpha_{1}^{\prime}=0, \beta_{1}^{\prime}=11.33, x_{2}=0$, $\alpha_{2}=1.3, \beta_{2}=0, \alpha_{2}^{\prime}=1.3$, and $\beta_{2}^{\prime}=0$.

Step 7: substituting the values of $x_{1}, \alpha_{1}, \beta_{1}$, $\alpha_{1}^{\prime}, \beta_{1}^{\prime}, x_{2}, \alpha_{2}, \beta_{2}, \alpha_{2}^{\prime}$, and $\beta_{2}^{\prime}$ in $X_{1}=\left(x_{1} ; \alpha_{1}, \beta_{1} ; \alpha_{1}^{\prime}, \beta_{1}^{\prime}\right)_{L R}$ and $X_{2}=\left(x_{2} ; \alpha_{2}, \beta_{2} ; \alpha_{2}^{\prime}, \beta_{2}^{\prime}\right)_{L R}$, the exact LR-type Pythagorean fuzzy optimal solution is $X_{1}=(9$; $0,8.8 ; 0,11.33)_{L R}$ and $X_{2}=(0 ; 1.3,0 ; 1.3,0)_{L R}$.

Step 8: substituting the values of $X_{1}$ and $X_{2}$, obtained in Step 7 into the objective function, the $L R$-type Pythagorean fuzzy optimal value is $(36 ; 16.8$, $70.8 ; 37.4,126.64)_{L R}$.
So, according to this technique, the farmer should grow $(9 ; 0,8.8 ; 0,11.33)_{L R}$ number of plants of $X$ and $(0 ; 1.3,0 ; 1.3,0)_{L R}$ number of plants of $Y$ to get a maximum profit of $(36 ; 16.8,70.8 ; 37.4,126.64)_{L R}$.

Example 2. Let us solve the practical model, discussed in [15], by method 1 as discussed in Section 3.1, using $L(x)=$ $R(x)=\max \{0,1-x\}$ and $L^{\prime}(x)=R^{\prime}(x)=\min \{1, x\}$ :

$$
\begin{gathered}
\operatorname{Max}\left(8 ; 2,2 ; \frac{5}{2}, \frac{5}{2}\right)_{L R} \otimes X_{1} \oplus\left(12 ; 2,2 ; \frac{5}{2}, \frac{5}{2}\right)_{L R} \\
\otimes X_{2} \oplus\left(1 ; \frac{1}{4}, \frac{1}{4} ; \frac{1}{2}, \frac{1}{2}\right)_{L R} \otimes X_{3},
\end{gathered}
$$

which subject to

$$
\begin{gathered}
\left(5 ; \frac{1}{2}, \frac{1}{2} ; \frac{3}{4}, \frac{3}{4}\right)_{L R} \otimes X_{1} \oplus\left(5 ; \frac{1}{2}, \frac{1}{2} ; \frac{3}{4}, \frac{3}{4}\right)_{L R} \otimes X_{2} \oplus X_{3}=(155 ; 45,52 ; 60,70)_{L R} \\
\left(6 ; \frac{1}{4}, \frac{1}{4} ; \frac{1}{2}, \frac{1}{2}\right)_{L R} \otimes X_{1} \oplus\left(2 ; \frac{1}{4}, \frac{1}{4} ; \frac{1}{2}, \frac{1}{2}\right)_{L R} \otimes X_{2} \preccurlyeq(125 ; 18,22 ; 30,35)_{L R}, \\
\left(1 ; \frac{1}{2}, \frac{1}{4} ; \frac{3}{4}, \frac{1}{2}\right)_{L R} \otimes X_{1} \oplus\left(4 ; \frac{1}{4}, \frac{1}{2} ; \frac{1}{2}, \frac{1}{4}\right)_{L R} \otimes X_{2} \preccurlyeq(110 ; 42,38 ; 65,60)_{L R},
\end{gathered}
$$

where $X_{j}$ are $L R$-type PFNs for $j=1,2,3$.

Let $X_{1}=\left(x_{1} ; \alpha_{1}, \beta_{1} ; \alpha_{1}^{\prime}, \beta_{1}^{\prime}\right)_{L R}, \quad X_{2}=\left(x_{2} ; \alpha_{2}, \beta_{2} ; \alpha_{2}^{\prime}\right.$, $\left.\beta_{2}^{\prime}\right)_{L R}, X_{3}=\left(x_{3} ; \alpha_{3}, \beta_{3} ; \alpha_{3}^{\prime}, \beta_{3}^{\prime}\right)_{L R}, \quad S_{1}=\left(m_{1} ; e_{1}, f_{1} ; g_{1}, h_{1}\right)_{L R}$,
$S_{2}=\left(m_{2} ; e_{2}, f_{2} ; g_{2}, h_{2}\right)_{L R}, \quad S_{1}^{\prime}=\left(m_{1}^{\prime} ; e_{1}^{\prime}, f_{1}^{\prime} ; g_{1}^{\prime}, h_{1}^{\prime}\right)_{L R}$, and $S_{2}^{\prime}=\left(m_{2}^{\prime} ; e_{2}^{\prime}, f_{2}^{\prime} ; g_{2}^{\prime}, h_{2}^{\prime}\right)_{L R}$. By solving step by step as discussed in Section 3.1, we obtain the crisp problem as given under 


$$
\begin{aligned}
\operatorname{Max} & \left(8 x_{1}+12 x_{2}+x_{3}-\alpha_{1}-\frac{1}{4}\left|x_{1}-\alpha_{1}\right|-\frac{3}{2} \alpha_{2}-\frac{1}{4}\left|x_{2}-\alpha_{2}\right|-\frac{1}{8} \alpha_{3}-\frac{1}{32}\left|x_{3}-\alpha_{3}\right|+\beta_{1}+\frac{1}{4}\left|x_{1}+\beta_{1}\right|\right. \\
& +\frac{3}{2} \beta_{2}+\frac{1}{4}\left|x_{2}+\beta_{2}\right|+\frac{1}{8} \beta_{3}+\frac{1}{32}\left|x_{3}+\beta_{3}\right|-\alpha_{1}^{\prime}-\frac{5}{16}\left|x_{1}-\alpha_{1}^{\prime}\right|-\frac{3}{2} \alpha_{2}^{\prime}-\frac{5}{16} \\
& \left.\left|x_{2}-\alpha_{2}^{\prime}\right|-\frac{1}{8} \alpha_{3}^{\prime}-\frac{1}{16}\left|x_{3}-\alpha_{3}^{\prime}\right|+\beta_{1}^{\prime}+\frac{5}{16}\left|x_{1}+\beta_{1}^{\prime}\right|+\frac{3}{2} \beta_{2}^{\prime}+\frac{5}{16}\left|x_{2}+\beta_{2}^{\prime}\right|+\frac{1}{2} \beta_{3}^{\prime}+\frac{1}{16}\left|x_{3}+\beta_{3}^{\prime}\right|\right),
\end{aligned}
$$

which subject to

$$
\begin{aligned}
& 5 x_{1}+5 x_{2}+x_{3}=155 \\
& 6 x_{1}+2 x_{2}+m_{1}=125+m_{1}^{\prime} \text {, } \\
& x_{1}+4 x_{2}+m_{2}=110+m_{2}^{\prime} \\
& 5 \alpha_{1}+\frac{1}{2}\left|x_{1}-\alpha_{1}\right|+5 \alpha_{2}+\frac{1}{2}\left|x_{2}-\alpha_{2}\right|+\alpha_{3}=45 \\
& 5 \beta_{1}+\frac{1}{2}\left|x_{1}+\beta_{1}\right|+5 \beta_{2}+\frac{1}{2}\left|x_{2}+\beta_{2}\right|+\beta_{3}=52, \\
& 5 \alpha_{1}^{\prime}+\frac{3}{4}\left|x_{1}-\alpha_{1}^{\prime}\right|+5 \alpha_{2}^{\prime}+\frac{3}{4}\left|x_{2}-\alpha_{2}^{\prime}\right|+\alpha_{3}^{\prime}=60, \\
& 5 \beta_{1}^{\prime}+\frac{3}{4}\left|x_{1}+\beta_{1}^{\prime}\right|+5 \beta_{2}^{\prime}+\frac{3}{4}\left|x_{2}+\beta_{2}^{\prime}\right|+\beta_{3}^{\prime}=70 \\
& 6 \alpha_{1}+\frac{1}{4}\left|x_{1}-\alpha_{1}\right|+2 \alpha_{2}+\frac{1}{4}\left|x_{2}-\alpha_{2}\right|+e_{1}=18+e_{1}^{\prime}, \\
& 6 \beta_{1}+\frac{1}{4}\left|x_{1}+\beta_{1}\right|+2 \beta_{2}+\frac{1}{4}\left|x_{2}+\beta_{2}\right|+f_{1}=22+f_{1}^{\prime} \text {, } \\
& 6 \alpha_{1}^{\prime}+\frac{1}{2}\left|x_{1}-\alpha_{1}^{\prime}\right|+2 \alpha_{2}^{\prime}+\frac{1}{2}\left|x_{2}-\alpha_{2}^{\prime}\right|+g_{1}=30+g_{1}^{\prime}, \\
& 6 \beta_{1}^{\prime}+\frac{1}{2}\left|x_{1}+\beta_{1}^{\prime}\right|+2 \beta_{2}^{\prime}+\frac{1}{2}\left|x_{2}+\beta_{2}^{\prime}\right|+h_{1}=35+h_{1}^{\prime} \\
& \frac{1}{8} x_{1}+\frac{7}{8} \alpha_{1}+\frac{3}{8}\left|x_{1}-\alpha_{1}\right|-\frac{1}{8} x_{2}+\frac{33}{8} \alpha_{2}+\frac{3}{8}\left|x_{2}-\alpha_{2}\right|+e_{2}=42+e_{2}^{\prime}, \\
& -\frac{1}{8} x_{1}+\frac{7}{8} \beta_{1}+\frac{3}{8}\left|x_{1}+\beta_{1}\right|+\frac{1}{8} x_{2}+\frac{33}{8} \beta_{2}+\frac{3}{8}\left|x_{2}+\beta_{2}\right|+f_{2}=38+f_{2}^{\prime} \text {, } \\
& \frac{1}{8} x_{1}+\frac{7}{8} \alpha_{1}^{\prime}+\frac{5}{8}\left|x_{1} \alpha_{1}^{\prime}\right|-\frac{1}{8} x_{2}+\frac{33}{8} \alpha_{2}^{\prime}+\frac{5}{8}\left|x_{2}-\alpha_{2}^{\prime}\right|+g_{2}=65+g_{2}^{\prime}, \\
& -\frac{1}{8} x_{1}+\frac{7}{8} \beta_{1}^{\prime}+\frac{5}{8}\left|x_{1}+\beta_{1}^{\prime}\right|+\frac{1}{8} x_{2}+\frac{33}{8} \beta_{2}^{\prime}+\frac{5}{8}\left|x_{2}+\beta_{2}^{\prime}\right|+h_{2}=60+h_{2}^{\prime},
\end{aligned}
$$

$8 m_{1}-e_{1}+f_{1}-g_{1}+h_{1}-8 m_{1}^{\prime}+e_{1}^{\prime}-f_{1}^{\prime}+g_{1}^{\prime}-h_{1}^{\prime} \geq 0,8 m_{2}-$ $e_{2}+f_{2}-g_{2}+h_{2}-8 m_{2}^{\prime}+e_{2}^{\prime}-f_{2}^{\prime}+g_{2}^{\prime}-h_{2}^{\prime} \geq 0, \quad \alpha_{1} \geq 0, \beta_{1} \geq$ $0, \alpha_{1}^{\prime}-\alpha_{1} \geq 0, \beta_{1}^{\prime}-\beta_{1} \geq 0, \alpha_{2} \geq 0, \beta_{2} \geq 0, \alpha_{2}^{\prime}-\alpha_{2} \geq 0, \beta_{2}^{\prime}-\beta_{2} \geq$
$0, e_{1} \geq 0, \quad f_{1} \geq 0, g_{1}-e_{1} \geq 0, h_{1}-f_{1} \geq 0, e_{1}^{\prime} \geq 0, f_{1}^{\prime} \geq 0, \quad g_{1}^{\prime}-$ $e_{1}^{\prime} \geq 0, h_{1}^{\prime}-f_{1}^{\prime} \geq 0, e_{2} \geq 0, f_{2} \geq 0, g_{2}-e_{2} \geq 0, h_{2}-f_{2} \geq 0, e_{2}^{\prime}$ $\geq 0, f_{2}^{\prime} \geq 0, g_{2}^{\prime}-e_{2}^{\prime} \geq 0$, and $h_{2}^{\prime}-f_{2}^{\prime} \geq 0$. 


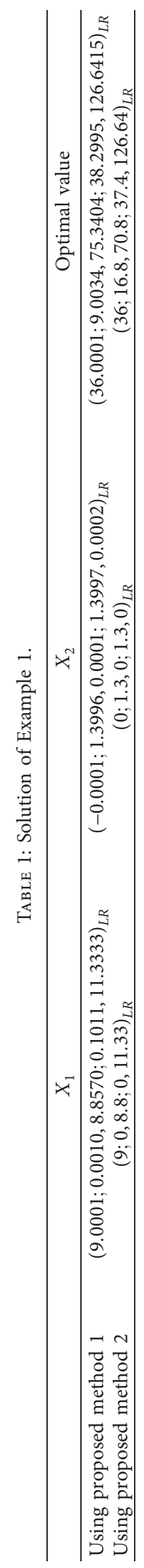




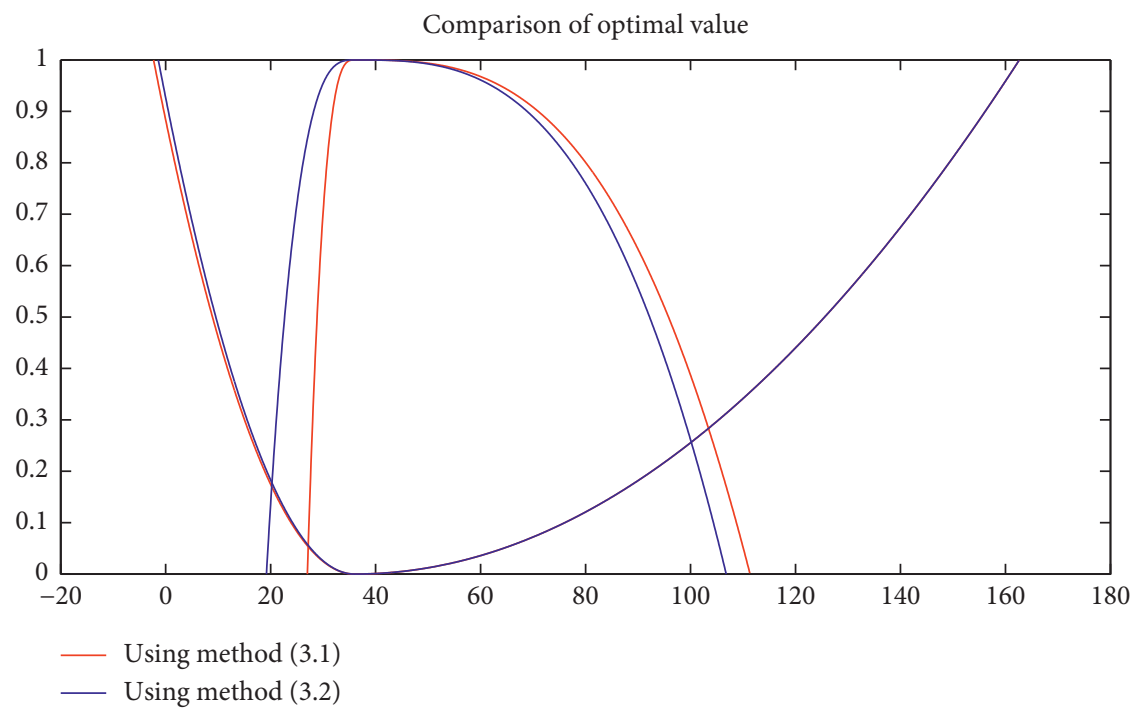

Figure 1: Comparison of the Pythagorean fuzzy optimal value using proposed methods (method 1 as discussed in Section $3.1=$ red and method 2 as discussed in Section 3.2=blue).

The optimal solution of this nonlinear problem (using MATLAB R2014a, solver "fmincon," algorithm “interior point," TolFun $=0.1$, TolX $=$ eps, TolCon $=0.1)$ is $X_{1}=$ $(12.9809,0.0332,0.0866 ; 0.0749,0.1206)_{L R}, X_{2}=(23.8686$; $4.4142,3.0225 ; 5.8714,4.3456)_{L R}$, and $X_{3}=(-29.2479$; $6.5785,16.4640 ; 7.0914,16.6821)_{L R}$. The optimal value of this problem is $(368.27 ; 117.03,109.26 ; 159.43,146.96)_{L R}$.

\section{Comparison with Existing Linear Programming Model}

Perez-Cañedo et al. [15] developed a method to solve $L R$-type fully intuitionistic fuzzy linear programming model. We have proposed two methods to solve $L R$-type FPFLPP with mixed constraints. By applying the proposed methods to Example 1, we have obtained the solution. Results of Example 1 are given in Table 1 and are shown graphically in Figure 1. Furthermore, we have solved the practical model [15] by using $L(x)=R(x)=\max \{0,1-x\}$ and $L^{\prime}(x)=R^{\prime}(x)=\min \{1, x\}$, and results are given in Table 2. Solution with existing method [15] with permutation $(\mathcal{S}, A, M, D, E)$ and solution with method 1 as discussed in Section 3.1 are compared in Figure 2. We observe the following facts:

(1) Our proposed methods are equivalent in terms of handling the inequality constraints of FPFLPP with $L R$-type PFNs as variables and parameters.

(2) Example 1 is solved by using our proposed methods. We see from Table 1 and Figure 1 that both methods produce the optimal solution which is almost the same.

(3) The solutions of both the methods (method 1 and method 2) are obtained by solving ultimately a crisp linear programming problem, which is mostly done using any software. The iterations needed for the solution of the crisp problem may vary problem to problem and may also depend on one of the methods used.

(4) We compare the solutions of our proposed method 1 with the existing method [15]. We see from Table 2 and Figure 2 that both the solutions are consistent to a large extent.

\section{Merits of the Proposed Methods}

The proposed mathematical model is based on the Pythagorean fuzzy environment. The advantages of the proposed method as compared to the existing method are as follows:

(1) There is no method to solve FPFLPP in which all the variables and parameters are unrestricted $L R$-type PFNs. Thus, this contribution is new and very helpful for the decision makers.

(2) A Pythagorean fuzzy model is more powerful than an intuitionistic fuzzy model since the intuitionistic fuzzy model cannot handle the situation where sum of membership degree $\mu$ and nonmembership degree $v$ of an element exceed 1. So, these techniques are more general and can be used in an intuitionistic fuzzy environment or fuzzy environment.

(3) The proposed techniques give almost the same results, so these techniques can be used depending on the interest of the decision maker.

Apart from all the benefits, the presented methods have some limitations. Our proposed methods fail where the condition $\mu^{2}+v^{2}>1$. 


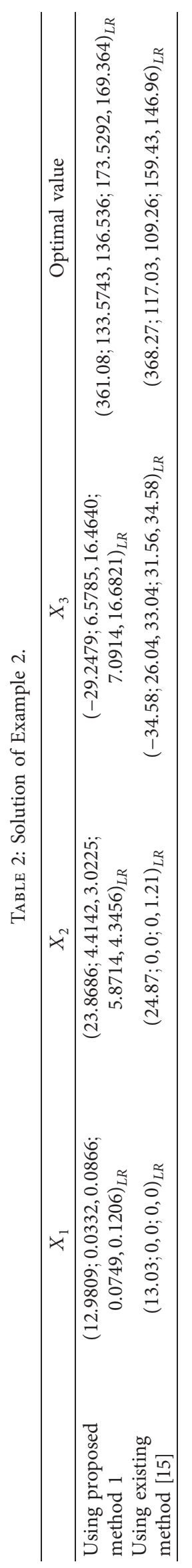




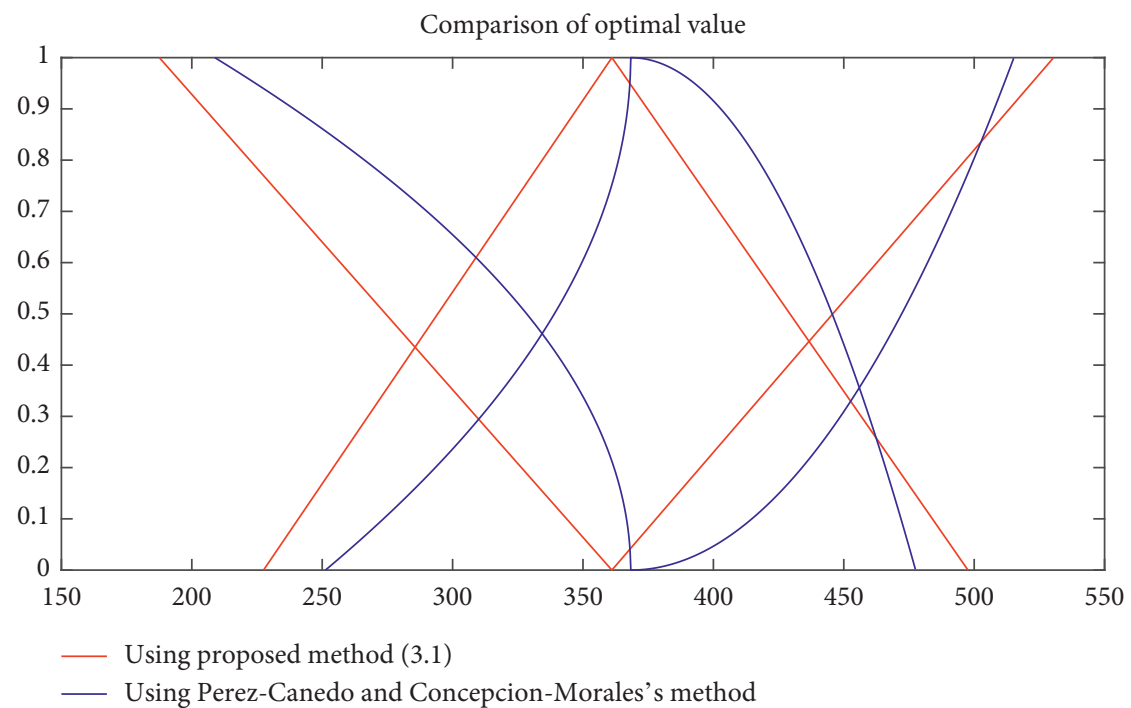

FIGURE 2: Comparison of optimal value using the existing method [15] is in blue and using method 1, as discussed in Section 3.1, is in red.

\section{Conclusions and Future Directions}

In mathematical programming models, linear programming problems are the simplest and most extensively used model. The linear programming model is easily applicable to various real-life applications. In this article, we have studied two techniques to solve $L R$-type FPFLPP with mixed constraints. We have shown the equivalence of both the presented methods. We have compared the results obtained from both the proposed techniques which come out to be almost the same. Furthermore, we have compared with the existing method [15]. In the future, our research work can be extended for nonlinear programming problems, fractional programming problems, and transportation problems.

\section{Data Availability}

No data were used to support this study.

\section{Ethical Approval}

This article does not contain any studies with human participants or animals performed by any of the authors.

\section{Conflicts of Interest}

The authors declare that they have no conflicts of interest.

\section{References}

[1] L. A. Zadeh, "Fuzzy sets," Information and Control, vol. 8, no. 3, pp. 338-353, 1965.

[2] L. A. Zadeh, "The concept of a linguistic variable and its application to approximate reasoning-I," Information Sciences, vol. 8, no. 3, pp. 199-249, 1975.

[3] R. E. Bellman and L. A. Zadeh, "Decision making in a fuzzy environment," Management Science, vol. 17, pp. 141-164, 1970.
[4] H.-J. Zimmermann, "Fuzzy programming and linear programming with several objective functions," Fuzzy Sets and Systems, vol. 1, no. 1, pp. 45-55, 1978.

[5] H. Tanaka $\dagger$, T. Okuda, and K. Asai, "On fuzzy-mathematical programming," Journal of Cybernetics, vol. 3, no. 4, pp. 37-46, 1973.

[6] T. Allahviranloo, "The adomian decomposition method for fuzzy system of linear equations," Applied Mathematics and Computation, vol. 163, no. 2, pp. 553-563, 2005.

[7] T. Allahviranloo, F. H. Lotfi, M. K. Kiasary, N. A. Kiani, and L. A. Zadeh, "Solving fully fuzzy linear programming problem by the ranking function," Applied Mathematical Sciences, vol. 2, no. 1, pp. 19-32, 2008.

[8] F. Hosseinzadeh Lotfi, T. Allahviranloo, M. Alimardani Jondabeh, and L. Alizadeh, "Solving a full fuzzy linear programming using lexicography method and fuzzy approximate solution," Applied Mathematical Modelling, vol. 33, no. 7, pp. 3151-3156, 2009.

[9] A. Kumar, J. Kaur, and P. Singh, "A new method for solving fully fuzzy linear programming problems," Applied Mathematical Modelling, vol. 35, no. 2, pp. 817-823, 2011.

[10] J. Kaur and A. Kumar, "Exact fuzzy optimal solution of fully fuzzy linear programming problems with unrestricted fuzzy variables," Applied Intelligence, vol. 37, no. 1, pp. 145-154, 2012.

[11] J. Kaur and A. Kumar, "Mehar's method for solving fully fuzzy linear programming problems with L-R fuzzy parameters," Applied Mathematical Modelling, vol. 37, no. 12-13, pp. 7142-7153, 2013.

[12] S. Moloudzadeh, T. Allahviranloo, and P. Darabi, "A new method for solving an arbitrary fully fuzzy linear system," Soft Computing, vol. 17, no. 9, pp. 1725-1731, 2013.

[13] D. Behera, K. Peters, S. A. Edalatpanah, and D. Qiu, "New methods for solving imprecisely defined linear programming problem under trapezoidal fuzzy uncertainty," Journal of Information and Optimization Sciences, vol. 42, no. 3, pp. 603-629, 2021.

[14] H. S. Najafi and S. A. Edalatpanah, "A note on "a new method for solving fully fuzzy linear programming problems," Applied Mathematical Modelling, vol. 37, no. 14-15, pp. 7865-7867, 2013. 
[15] B. Perez-Canedo and E. R. Concepcion-Morales, "On-LRtype fully intuitionistic fuzzy linear programming with inequality constraints: solutions with unique optimal values," Expert Systems with Applications, vol. 128, pp. 246-255, 2019.

[16] K. T. Atanassov, "Intuitionistic fuzzy sets," Fuzzy Sets and Systems, vol. 20, no. 1, pp. 87-96, 1986.

[17] P. P. Angelov, "Optimization in an intuitionistic fuzzy environment," Fuzzy Sets and Systems, vol. 86, no. 3, pp. 299-306, 1997.

[18] D. Dubey and A. Mehra, "Linear programming with triangular intuitionistic fuzzy number," Advances in Intelligent Systems Research, vol. 1, no. 1, pp. 563-569, 2011.

[19] R. Parvathi and C. Malathi, "Intuitionistic fuzzy linear programming problems World," Applied Sciences Journal, vol. 17, no. 12, pp. 1787-1791, 2012.

[20] A. Nagoorgani and K. Ponnalagu, "A new approach on solving intuitionistic fuzzy linear programming problem," Applied Mathematical Sciences, vol. 6, no. 70, pp. 3467-3474, 2012.

[21] R. Parvathi and C. Malathi, "Intuitionistic fuzzy linear optimization," Notes on Intuitionistic Fuzzy Sets, vol. 18, no. 1, pp. 48-56, 2012.

[22] R. Parvathi, C. Malathi, M. Akram, and K. T. Atanassov, "Intuitionistic fuzzy linear regression analysis," Fuzzy Optimization and Decision Making, vol. 12, no. 2, pp. 215-229, 2013.

[23] H. Garg, M. Rani, S. P. Sharma, and Y. Vishwakarma, "Intuitionistic fuzzy optimization technique for solving multiobjective reliability optimization problems in interval environment," Expert Systems with Applications, vol. 41, no. 7, pp. 3157-3167, 2014.

[24] M. Suresh, S. Vengataasalam, and K. Arun Prakash, "Solving intuitionistic fuzzy linear programming problems by ranking function," Journal of Intelligent \& Fuzzy Systems, vol. 27, no. 6, pp. 3081-3087, 2014.

[25] A. Nagoorgani, J. Kavikumar, and K. Ponnalagu, "The knowledge of expert opinion in intuitionistic fuzzy linear programming problem," Mathematical Problems in Engineering, vol. 2015, Article ID 875460, 7 pages, 2015.

[26] V. Singh and S. P. Yadav, "Development and optimization of unrestricted LR-type intuitionistic fuzzy mathematical programming problems," Expert Systems With Applications, vol. 80, pp. 147-161, 2017.

[27] S. K. Singh and S. P. Yadav, "Intuitionistic fuzzy multiobjective linear programming problem with various membership functions," Annals of Operations Research, vol. 269, no. 1-2, pp. 693-707, 2018.

[28] C. Malathi and P. Umadevi, "A new procedure for solving linear programming problems in an intuitionistic fuzzy environment," Journal of Physics: Conference Series, vol. 1139, Article ID 012079, 2018.

[29] S. Abhishekh and A. K. Nishad, "A novel ranking approach to solving fully $L R$-intuitionistic fuzzy transportation problem," New Mathematics and Natural Computation, vol. 15, no. 1, pp. 95-112, 2019.

[30] S. K. Bharati and S. R. Singh, "Solution of multiobjective linear programming problems in interval-valued intuitionistic fuzzy environment," Soft Computing, vol. 23, no. 1, pp. 77-84, 2019.

[31] A. Kabiraj, P. K. Nayak, and S. Raha, "Solving intuitionistic fuzzy linear programming problem," International Journal of Intelligence Science, vol. 9, no. 1, pp. 44-58, 2019.

[32] R. R. Yager, "Pythagorean fuzzy subsets," in Proceedings of the Joint IFSA World Congress and NAFIPS Annual Meeting, pp. 57-61, Edmonton, Canada, August 2013.
[33] R. R. Yager and A. M. Abbasov, "Pythagorean membership grades, complex numbers, and decision making," International Journal of Intelligent Systems, vol. 28, no. 5, pp. 436452, 2013.

[34] R. R. Yager, "Pythagorean membership grades in multicriteria decision making," IEEE Transactions on Fuzzy Systems, vol. 22, no. 4, pp. 958-965, 2014.

[35] X. Zhang and Z. Xu, "Extension of TOPSIS to multiple criteria decision making with pythagorean fuzzy sets," International Journal of Intelligent Systems, vol. 29, no. 12, pp. 1061-1078, 2014.

[36] X. Peng, H. Yuan, and Y. Yang, "Pythagorean fuzzy information measures and their applications," International Journal of Intelligent Systems, vol. 32, no. 10, pp. 991-1029, 2017.

[37] S.-P. Wan, Z. Jin, and J.-Y. Dong, "Pythagorean fuzzy mathematical programming method for multi-attribute group decision making with Pythagorean fuzzy truth degrees," Knowledge and Information Systems, vol. 55, no. 2, pp. 437-466, 2018.

[38] R. Kumar, S. A. Edalatpanah, S. Jha, and R. Singh, "A Pythagorean fuzzy approach to the transportation problem," Complex \& Intelligent Systems, vol. 5, no. 2, pp. 255-263, 2019.

[39] A. Luqman, M. Akram, and J. C. R. Alcantud, "Digraph and matrix approach for risk evaluations under Pythagorean fuzzy information," Expert Systems with Applications, vol. 43, Article ID 114518, 2020.

[40] S.-P. Wan, Z. Jin, and J.-Y. Dong, "A new order relation for Pythagorean fuzzy numbers and application to multi-attribute group decision making," Knowledge and Information Systems, vol. 62, no. 2, pp. 751-785, 2020.

[41] F. Ahmad and A. Y. Adhami, "Spherical fuzzy linear programming problem," in Decision Making with Spherical Fuzzy Sets. Studies in Fuzziness and Soft Computing, C. Kahraman and F. Kutlu Gndogdu, Eds., p. 392, Springer, Berlin, Germany, 2021.

[42] M. Akram, I. Ullah, T. Allahviranloo, and S. A. Edalatpanah, "Fully Pythagorean fuzzy linear programming problems with equality constraints," Computational and Applied Mathematics, vol. 40, no. 4, p. 120, 2021.

[43] C. Wei, J. Wu, Y. Guo, and G. Wei, “Green supplier selection based on CODAS method in probabilistic uncertain linguistic environment," Technological and Economic Development of Economy, vol. 27, no. 3, pp. 530-549, 2021.

[44] G. Wei, J. Wu, Y. Guo, J. Wang, and C. Wei, “An extended COPRAS model for multiple attribute group decision making based on single-valued neutrosophic 2-tuple linguistic environment," Technological and Economic Development of Economy, vol. 27, no. 2, pp. 353-368, 2021.

[45] Y. Zhang, G. Wei, Y. Guo, and C. Wei, "TODIM method based on cumulative prospect theory for multiple attribute group decision-making under 2-tuple linguistic Pythagorean fuzzy environment," International Journal of Intelligent Systems, vol. 36, no. 6, pp. 2548-2571, 2021.

[46] M. Akram, I. Ullah, T. Allahviranloo, and S. A. Edalatpanah, "LR-type fully pythagorean fuzzy linear programming problems with equality constraints," Journal of Intelligent \& Fuzzy Systems, vol. 13, pp. 1-18, 2021.

[47] M. Akram, T. Allahviranloo, W. Pedrycz, and M. Ali, "Methods for solving LR-bipolar fuzzy linear systems," Soft Computing, vol. 25, no. 1, pp. 85-108, 2021.

[48] J.-J. An and D.-F. Li, "A linear programming approach to solve constrained bi-matrix games with intuitionistic fuzzy 
payoffs," International Journal of Fuzzy Systems, vol. 21, no. 3, pp. 908-915, 2019.

[49] T.-Y. Chen, "Pythagorean fuzzy linear programming technique for multidimensional analysis of preference using a squared-distance-based approach for multiple criteria decision analysis," Expert Systems with Applications, vol. 164, Article ID 113908, 2021.

[50] S. A. Edalatpanah, "A data envelopment analysis model with triangular intuitionistic fuzzy numbers," International Journal of Data Envelopment Analysis, vol. 7, no. 4, pp. 47-58, 2019.

[51] J. Kaur and A. Kumar, An Introduction to Fuzzy Linear Programming Problems, Springer Science and Business Media LLC, Berlin, Germany, 2016.

[52] G. S. Mahapatra and T. K. Roy, "Reliability evaluation using triangular intuitionistic fuzzy numbers arithmetic operations," World Acadmy of Science, Engineering and Technology, vol. 50, pp. 574-581, 2009.

[53] G. S. Mahapatra and T. K. Roy, "Intuitionistic fuzzy number and its arithmetic operation with application on system failure," Journal of Uncertain Systems, vol. 7, no. 2, pp. 92-107, 2013.

[54] H. Saberi Najafi, S. A. Edalatpanah, and H. Dutta, "A nonlinear model for fully fuzzy linear programming with fully unrestricted variables and parameters," Alexandria Engineering Journal, vol. 55, no. 3, pp. 2589-2595, 2016.

[55] X. Peng and Y. Yang, "Some results for Pythagorean fuzzy sets," International Journal of Intelligent Systems, vol. 30, no. 11, Article ID 11331160, 2015.

[56] M. Saqib, M. Akram, S. Bashir, and T. Allahviranloo, "Numerical solution of bipolar fuzzy initial value problem," Journal of Intelligent \& Fuzzy Systems, vol. 40, no. 1, pp. 1309-1341, 2021.

[57] W. Xue, Z. Xu, X. Zhang, and X. Tian, "Pythagorean fuzzy LINMAP method based on the entropy theory for railway project investment decision making," International Journal of Intelligent Systems, vol. 33, no. 1, pp. 93-125, 2018. 